\title{
Cardioprotection of dapagliflozin and vildagliptin in rats with cardiac ischemia-reperfusion injury
}

\author{
Pongpan Tanajak1,2,3, Piangkwan Sa-nguanmoo1,2,3, Sivaporn Sivasinprasasn1, Savitree Thummasorn1,2,3, \\ Natthaphat Siri-Angkul1,2,3, Siriporn C Chattipakorn1,3,4 and Nipon Chattipakorn1,2,3 \\ ${ }^{1}$ Cardiac Electrophysiology Research and Training Center, Faculty of Medicine, Chiang Mai University, Chiang Mai, Thailand \\ ${ }^{2}$ Cardiac Electrophysiology Unit, Department of Physiology, Faculty of Medicine, Chiang Mai University, Chiang Mai, Thailand \\ ${ }^{3}$ Center of Excellence in Cardiac Electrophysiology Research, Chiang Mai University, Chiang Mai, Thailand \\ ${ }^{4}$ Department of Oral Biology and Diagnostic Sciences, Faculty of Dentistry, Chiang Mai University, Chiang Mai, Thailand \\ Correspondence should be addressed to N Chattipakorn: nchattip@gmail.com
}

\section{Abstract}

Sodium-glucose cotransporter 2 inhibitor (SGLT2-i) effects on cardiac ischemia/ reperfusion (I/R) injury are unclear. Unlike SGLT2-i, dipeptidyl peptidase 4 inhibitors (DPP4-i) have shown effective cardioprotection in cardiac I/R injury. We aimed to investigate whether SGLT2-i reduces myocardial dysfunction and myocardial injury to a greater extent than DPP4-i in obese insulin-resistant rats with/without cardiac I/R injury. The high-fat (HF) diet-induced obese insulin-resistant rats were divided into 4 groups and received the following treatments for 28 days: vehicle (HFV); vildagliptin at a dosage of $3 \mathrm{mg} / \mathrm{kg} /$ day (HFVil); dapagliflozin at a dosage of $1 \mathrm{mg} / \mathrm{kg} /$ day (HFDa) and combination drugs (HFDaVil). At the end, I/R injury was induced by a 30-min left anterior descending coronary occlusion and 120-min reperfusion. Dapagliflozin showed a greater efficacy than vildagliptin in improving the metabolic impairments, low frequency/high frequency (LF/HF) ratio, systolic blood pressure and left ventricular (LV) function in comparison to HFV rats. In cardiac I/R injury, dapagliflozin had a greater efficacy than vildagiptin in decreasing mitochondrial DRP1, cleaved caspase 3, LV dysfunction and infarct size in comparison to HFV rats. However, the combined therapy showed the greatest efficacy in attenuating LV dysfunction, mitochondrial DRP1 and infarct size in comparison to HFV rats. In conclusion, dapagliflozin has a more pronounced effect than vildagliptin in obese insulin-resistant rats for the improvement of LV function. In rats with cardiac I/R injury, although dapagliflozin had a greater efficacy on cardioprotection than vildagliptin, the combined therapy exerted the highest cardioprotective effects potentially by reducing mitochondrial fission.

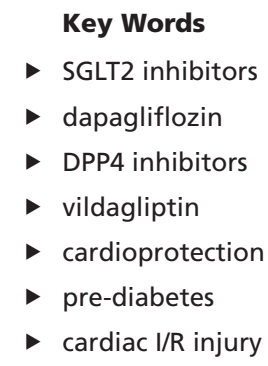

Journal of Endocrinology (2018) 236, 69-84

\section{Introduction}

The sodium-glucose cotransporter 2 inhibitors (SGLT2-i) are a new class of anti-diabetic drugs, which exhibit a blood glucose-lowering effect by inhibiting glucose reabsorption at the proximal tubule of the nephron, resulting in decreased renal glucose reabsorption and increased renal glucose excretion, thus improving glycemic control (Vrhovac et al. 2015). A recent clinical trial, EMPA-REG OUTCOME, demonstrated that SGLT2-i empagliflozin reduced cardiovascular morbidity and mortality in patients with type 2 diabetes (T2DM) who are at a high risk 
of cardiovascular events. Although the benefits of SGLT2-i are potentially through cardiometabolic regulation, this effect of SGLT2-i in comparison with other anti-diabetic drugs, especially in pre-diabetic conditions both with and without myocardial ischemia/reperfusion (I/R) injury, as well as its underlying mechanism have never been investigated.

Incretin-based therapy using dipeptidyl peptidase 4 inhibitors (DPP4-i) is a well-known effective glycemic control remedy. The cardioprotective effects of DPP4-i beyond its glycemic regulating effects have been reported to lead to an improvement in cardiac function in high-fat diet (HFD)-induced pre-diabetes rat models (Apaijai et al. 2013, Tanajak et al. 2017). Treatment with DPP4-i provided effective cardioprotection in rats with myocardial I/R injury (Chinda et al. 2014). In healthy volunteers, treatment with vildagliptin at the highest daily therapeutic dose or a fourfold higher dose did not change the QT interval, nor did it have adverse effects on cardiac conduction (He et al. 2011). However, the benefit of its glycemic control as well as cardioprotection in obese insulin-resistant with cardiac $\mathrm{I} / \mathrm{R}$ injury compared to SGLT2-i is not known.

This study aimed to investigate the effects of SGLT2-i (dapaglipflozin), DPP4-i (vildagliptin) and a combined therapy of both agents on metabolic regulation, blood chemistry, blood pressure, heart rate variability (HRV) and LV function in HFD- induced pre-diabetic rats. Moreover, the therapeutic effects of these drugs on cardiac arrhythmia, myocardial infarction size and LV function, as well as the underlying mechanisms under cardiac I/R injury were investigated in HFD-induced obese insulin-resistant rats. Our hypothesis is that dapaglipflozin provides more effective metabolic regulation and cardioprotection than vildagliptin in HFD-induced obese insulin-resistant rats, both with/without cardiac I/R injury and also that the combined therapy exerts superior cardioprotection in these rats, when compared with single regimens.

\section{Materials and methods}

\section{Ethical approval}

All experimental protocols in this study were approved by the Faculty of Medicine, Chiang Mai University Institutional Animal Care and Use Committee (Permit No. 46/2558), in compliance with NIH guidelines and in accordance with the ARRIVE guidelines for reporting experiments involving animals (Kilkenny et al. 2010).

\section{Experimental animals}

After 1 week of acclimatization, all rats were randomized into 2 groups and were given either ND $(n=12)$ or HFD $(n=48)$ for 12 weeks. At week 12, ND rats were fed continuously with ND and vehicle (NDV) for 28 days. At week 12, HFD rats were subdivided into 4 experimental groups ( $n=12 /$ group), and each group received one of the following treatments via oral gavage feeding for 28 days: (1) HFD rats received vehicle (HFV); (2) HFD rats received vildagliptin $3 \mathrm{mg} / \mathrm{kg} /$ day (HFVil); (3) HFD rats received dapagliflozin $1 \mathrm{mg} / \mathrm{kg} /$ day (HFDa) or (4) HFD rats received the combination of vildagliptin $3 \mathrm{mg} / \mathrm{kg} /$ day and dapagliflozin $1 \mathrm{mg} / \mathrm{kg} /$ day (HFDaVil). At the end of week 12 of the assigned diet treatment and again after 28 days of drug treatments, the metabolic parameters, blood chemistry, 24-h urine volume, urinary glucose excretion (UGE), 24-h UGE, blood pressure and HRV were investigated. Then, in vivo myocardial I/R were induced by left anterior descending (LAD) coronary artery ligation for $30 \mathrm{~min}$ followed by reperfusion for $120 \mathrm{~min}$. During the myocardial I/R procedure, lead II ECG was used to record arrhythmia parameter measurements. LV function was determined using a pressure-volume (P-V) loop at the beginning of the $\mathrm{I} / \mathrm{R}$ procedure (baseline) and throughout the cardiac I/R study. At the end of the cardiac I/R procedure, the heart was removed rapidly and then perfused with $20 \mathrm{~mL}$ cold $0.9 \%$ NSS. Finally, the removed hearts were used to assess the levels of myocardial infarction, cardiac mitochondrial function and protein expression. The myocardium was divided into the remote area and ischemic area for measurements of cardiac mitochondrial function, cardiac lipid peroxidation, tissue proteins expression, mitochondrial protein expression and myocardial infarction area. The experimental design, the number of rats and the details of data sets for analysis are listed in Fig. 1.

\section{Myocardial I/R surgical procedure}

Rats were anesthetized by an intramuscular injection of Zoletil (zolazepam and tiletamine) $50 \mathrm{mg} / \mathrm{kg}$ in combination with xylazine $3 \mathrm{mg} / \mathrm{kg}$ and were ventilated via a tracheotomy tube by Harvard Rodent Ventilator Model 683 (Harvard Apparatus, Holliston, MA, USA) (Pongkan et al. 2016). Lead II ECG was monitored continuously throughout the entire $\mathrm{I} / \mathrm{R}$ procedure. A left intercostal thoracotomy incision was performed, and then the LAD coronary artery was identified and ligated at approximately $2 \mathrm{~mm}$ distal to the origin of the left coronary, by a 5-0 silk suture to induce myocardial infarction. The LAD was 


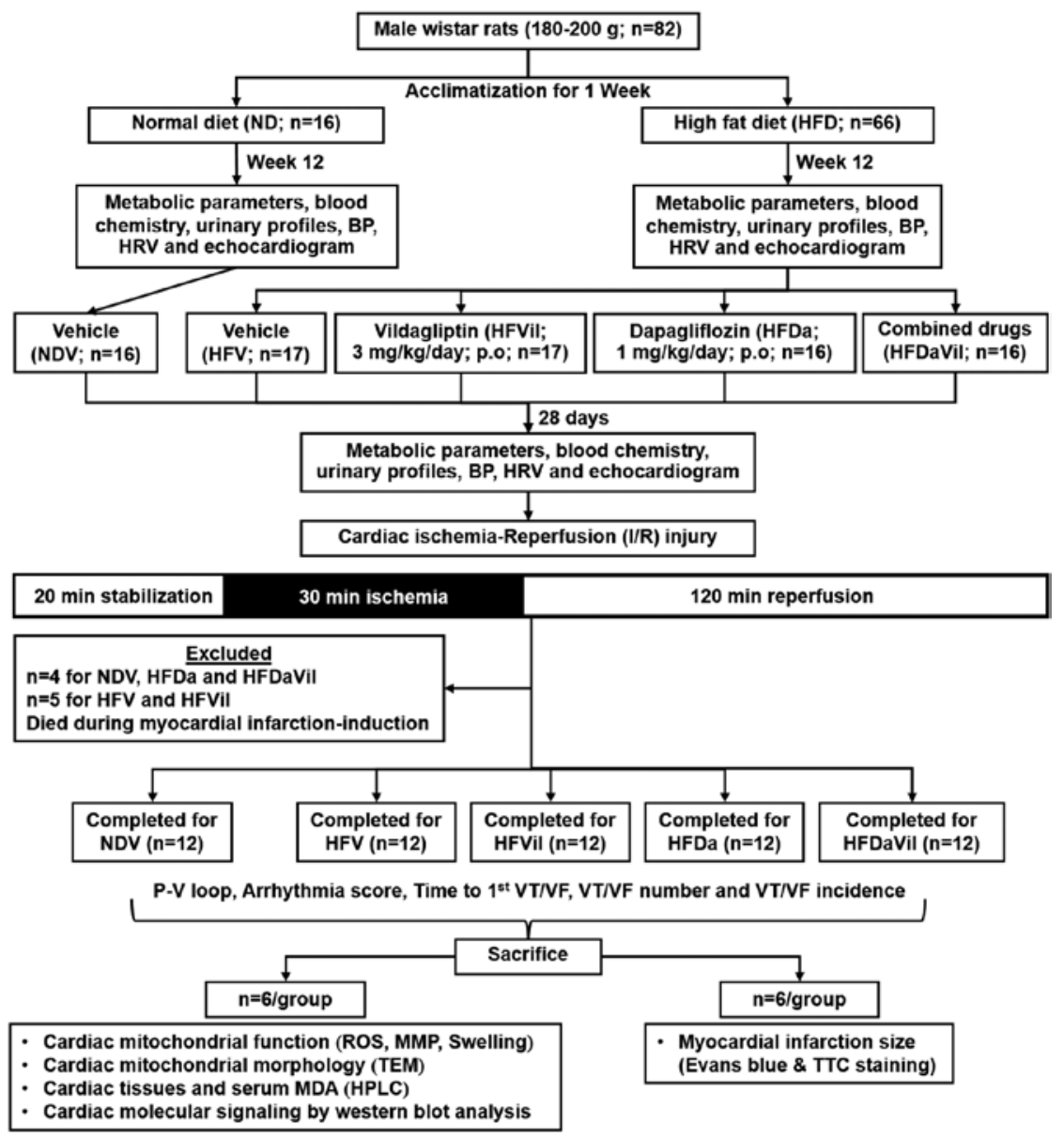

Figure 1

Diagram of the experimental protocol, number of animals and data set for analysis of this study. HFD, high-fat diet; HFDa, high-fat diet treated with dapagliflozin; HFDaVil, high-fat diet treated with vildagliptin and dapagliflozin; HFV, high-fat diet treated with vehicle; HFVil, high-fat diet treated with vildagliptin; HPLC, high-performance liquid chromatography; MMP, mitochondrial membrane potential; ND, normal diet; NDV, normal diet treated with vehicle; ROS, reactive oxygen species; TEM, transmission electron microscopy; TTC, triphenyltetrazolium chloride. ligated for $30 \mathrm{~min}$, the procedure being followed by $120 \mathrm{~min}$ of reperfusion. Myocardial ischemia was confirmed by ST elevation on the ECG recording (Pongkan et al. 2016).

\section{Pressure-volume (P-V) loop for LV function assessment during I/R}

During myocardial I/R study, an admittance-based P-V catheter (Transonic Scisense, Ontorio, Canada) was used to investigate LV function throughout the protocol. After the rats were anesthetized, the right common carotid artery (Rt. CCA) was identified. The P-V loop catheter was inserted into the Rt. CCA and advanced into the LV chamber for hemodynamic monitoring. The catheter was connected to the recording system operated by LabScribe 2 software (iWorx System, Dover, NH, USA) (Tanajak et al. $2016,2017)$. LV function parameters including heart rate (HR), end-systolic pressure (ESP), end-diastolic pressure (EDP), maximum pressure $\left(P_{\max }\right)$, minimum pressure $\left(P_{\min }\right), d P / d t_{\max }, d P / d t_{\min }$, stroke volume $(\mathrm{SV})$, ejection fraction (\%EF), end-systolic volume (ESV), end-diastolic volume (EDV) and stroke work (SW) were determined.

\section{Determination of arrhythmia parameters during cardiac I/R procedure}

The occurrence of cardiac arrhythmia was characterized using the Lambeth Conventions method (Curtis et al. 2013). Arrhythmia scores were determined using the criteria described in previous studies (Pongkan et al. 2015, 2016).

\section{Infarct size measurement}

At the end of the I/R experiment, the hearts were removed and irrigated with cold $0.9 \%$ NSS to wash out any residual blood from the chambers and great vessels. The LAD was re-occluded at the same site that was used previously during the ischemic period. The catheters were inserted into the aorta and retrogradely perfused with a 1\% Evans blue dye infusion. The area that could not be infused with the dye was defined as the area of no blood flow during the ischemic period. Then, the heart was frozen and cut horizontally into $1-\mathrm{mm}$ thick slices from the apex to $1 \mathrm{~mm}$ above the site of occlusion. The heart 
Table 1 The effects of vildagliptin, dapagliflozin and combined therapy on blood pressure in HFD rats after treatment for 4 weeks prior myocardial I/R injury.

\begin{tabular}{|c|c|c|c|c|c|}
\hline \multirow[b]{2}{*}{ Parameters } & \multicolumn{5}{|c|}{ Groups } \\
\hline & NDV & HFV & HFVil & HFDa & HFDaVil \\
\hline SBP (mmHg) & $\overline{126 \pm 4}$ & $147 \pm 3 *$ & $145 \pm 4 *$ & $\overline{118 \pm 5^{\dagger, \ddagger}}$ & $124 \pm 6^{+, \neq}$ \\
\hline $\mathrm{DBP}(\mathrm{mmHg})$ & $93 \pm 3$ & $98 \pm 2$ & $94 \pm 6$ & $91 \pm 5$ & $98 \pm 5$ \\
\hline $\mathrm{MAP}(\mathrm{mmHg})$ & $104 \pm 4$ & $126 \pm 4^{*}$ & $121 \pm 4^{*}$ & $105 \pm 3^{\dagger, \neq}$ & $105 \pm 3^{\dagger, \neq}$ \\
\hline
\end{tabular}

Data are shown as mean \pm S.E.M., $n=10-12$ /group.

${ }^{\star} P<0.05$ vs NDV; ${ }^{\dagger} P<0.05$ vs HFV; ${ }^{*} P<0.05$ vs HFVil.

DBP, diastolic blood pressure; HFDa, high-fat diet treated with dapagliflozin; HFDaVil, high-fat diet treated with dapagliflozin and vildagliptin; HFV, high-fat diet treated with vehicle; HFVil, high-fat diet treated with vildagliptin; MAP, mean arterial pressure; NDV, normal diet treated with vehicle; SBP, systolic blood pressure.

slices were incubated in $37^{\circ} \mathrm{C}, 1 \%$ triphenyltetrazolium chloride (TTC) for $15 \mathrm{~min}$ (Pongkan et al. 2016). After incubation, the area with viable tissue was red, whereas the area that did not stain with Evans blue was defined as the area at risk (AAR). The area that was neither blue nor red (white area) was defined as the infarct site (Riess et al. 2009, Chinda et al. 2014, Pongkan et al. 2015). The myocardial infarct size was measured using Image tools software, version 3.0 and was calculated, depending on the weight of each tissue slice in accordance with the formula by Reiss et al. (Riess et al. 2009, Chinda et al. 2014, Pongkan et al. 2015). The levels of myocardial infarct size were presented as a percentage of myocardial infarct size per AAR.

\section{Cardiac mitochondrial function and morphology}

\section{measurements}

After the cardiac I/R study, rats were killed, and the hearts were removed rapidly. The hearts were perfused with cold $0.9 \%$ NSS. Cardiac mitochondria were obtained from both remote and ischemic myocardium using a differential centrifugation technique as described previously (Thummasorn et al. 2011, Tanajak et al. 2017). Cardiac mitochondrial function, which is defined by the following parameters: cardiac mitochondrial reactive oxygen species (ROS), cardiac mitochondrial membrane potential change (red/green fluorescence intensity ratio) and cardiac mitochondrial swelling (absorbance intensity) were investigated. The details of the procedures for obtaining cardiac mitochondrial function and morphology measurements have been described previously (Tanajak et al. 2017). An increase in the fluorescent intensity indicates an increase in mitochondrial ROS production, which suggests increased oxidative stress levels. A decrease in the red/green fluorescence intensity ratio indicates an increase in cardiac mitochondrial membrane depolarization, whereas a decrease in mitochondrial absorbance indicates cardiac mitochondrial swelling (Thummasorn et al. 2011, Tanajak et al. 2017). Moreover, cardiac mitochondrial morphology was also demonstrated at $15,000 \times$ using a JEM-2200FS field emission electron microscope to confirm changes in mitochondrial structure or swelling.

\section{Plasma and cardiac lipid peroxidation assessments}

Blood was collected from the descending aorta after the rat was killed at the end of the myocardial I/R protocol, and then plasma was obtained and used for the study of lipid peroxidation levels. Tissue samples were obtained from both the remote and ischemic myocardium from the same heart that was used in the cardiac mitochondrial study. The homogenized heart tissues and plasma malondialdehyde (MDA) concentrations were determined using a high-performance liquid chromatography (HPLC)-based assay (Thermo Scientific) (Apaijai et al. 2013, Tanajak et al. 2017). Plasma and cardiac MDA were mixed with $\mathrm{H}_{3} \mathrm{PO}_{4}$ and thiobarbituric acid (TBA) to produce TBA-reactive substances (TBARS). Plasma and cardiac TBARS concentrations were determined directly from a standard curve and reported as equivalent to the MDA concentration. Cardiac MDA levels are presented as normalized units by protein concentration (Apaijai et al. 2013, Tanajak et al. 2017).

\section{Statistical analysis}

Data are reported as mean \pm s.E.M. in the tables and reported as medians and interquartile range in the graphs. Normal distribution of the data was assessed using the Kolmogorov-Sminov and Shapiro-Wilk normality test. Comparison between groups of the non-normally distributed data were performed using nonparametric statistical analysis (Kruskal-Wallis and Mann-Whitney) followed by a post hoc Bonferroni correction. Comparisons between groups for normally distributed data were performed using a one-way ANOVA followed by a post hoc Bonferroni correction. VT/VF incidence was compared among groups using a $\chi^{2}$ test. Data were analyzed using SPSS statistics software, version 22. All statistical tests conducted were two tailed, and results were considered to be statistically significant when the $P$ value was less than $0.05(P<0.05)$. 


\begin{tabular}{l|l|l|l|l|}
$\begin{array}{l}\text { Journal of } \\
\text { Endocrinology }\end{array}$ & P Tanajak et al. & $\begin{array}{l}\text { Dapagliflozin and vildagliptin in } \\
\text { the heart }\end{array}$ & $\mathbf{2 3 6 : 2}$ & $\mathbf{7 3}$ \\
\hline
\end{tabular}

\section{Results}

\section{Dapagliflozin provided better outcomes than}

vildagliptin in metabolic regulation, improved cardiac morphometric and urinary profiles in pre-diabetic rats

HFV rats had significantly decreased HDL-C levels and significantly increased body weight, visceral fat, heart weight, heart weight/body weight ratio, plasma insulin, HOMA index, AUCg, plasma TC, plasma LDL-C and plasma FGF21 levels, when compared with NDV rats
(Supplementary Table 1 , see section on supplementary data given at the end of this article). Regarding plasma TG levels, there was not changed in all groups (Supplementary Table 1). After treatment, all vildagliptin-treated rats had a decrease in plasma insulin, HOMA index, AUCg and plasma FGF21 levels, when compared with the HFV rats. Treatments with dapagliflozin and combined therapy showed a greater efficacy than vildagliptin in decreasing body weight, visceral fat, heart weight, heart weight/body weight ratio, plasma insulin, plasma glucose, HOMA index
A

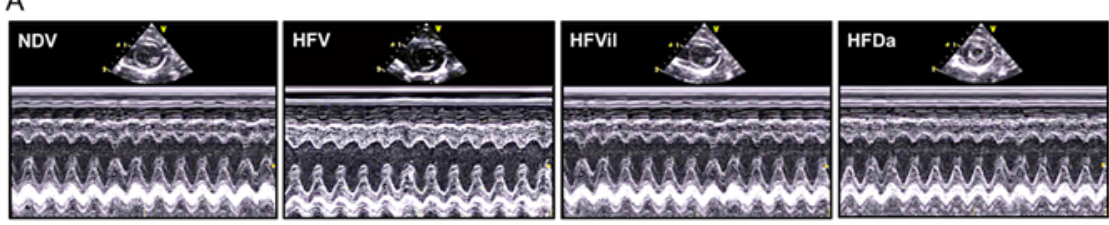

B
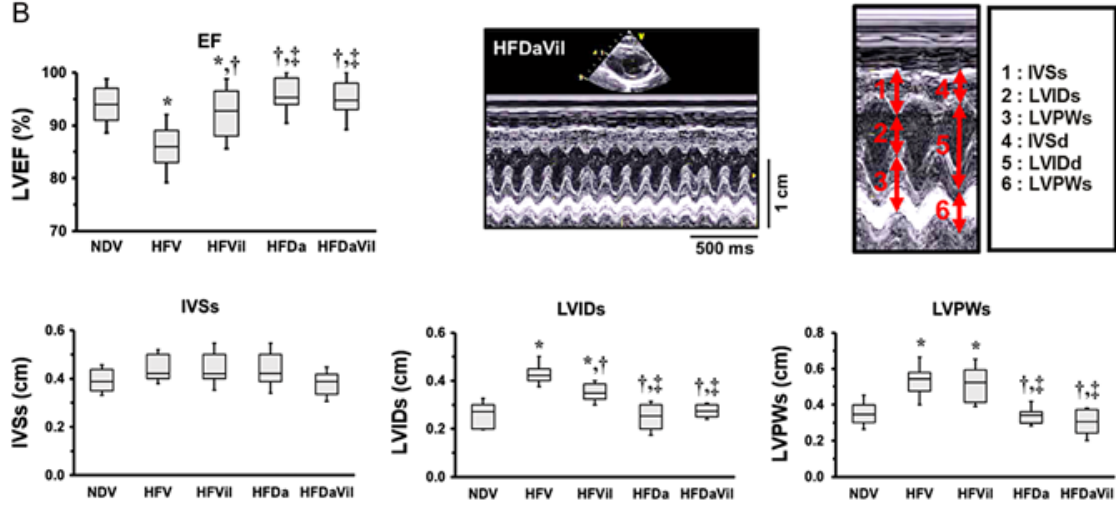

IVSd
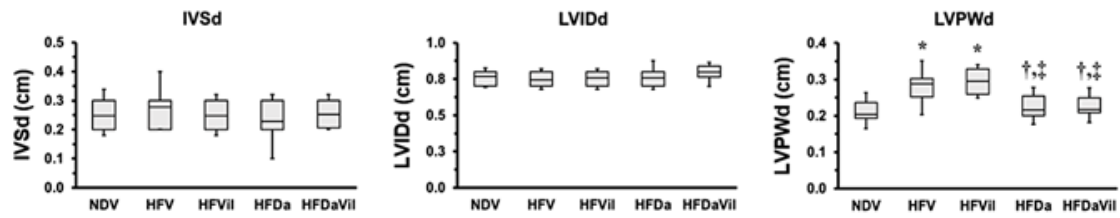

C
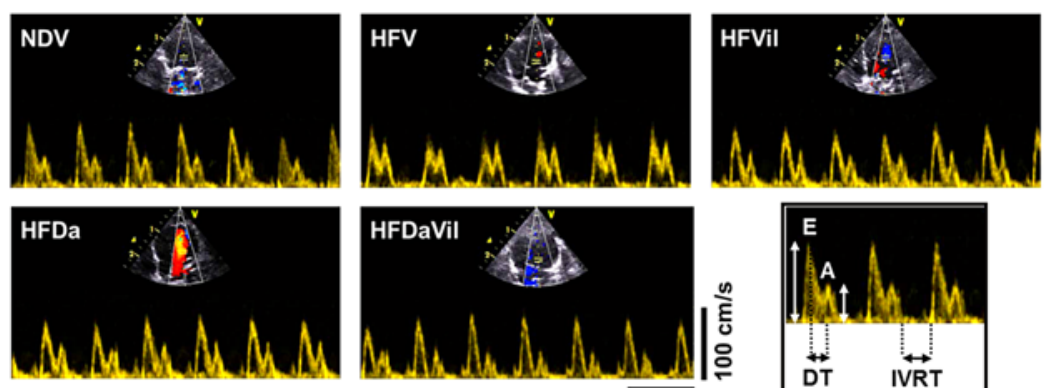

$\overline{200 \mathrm{~ms}}$
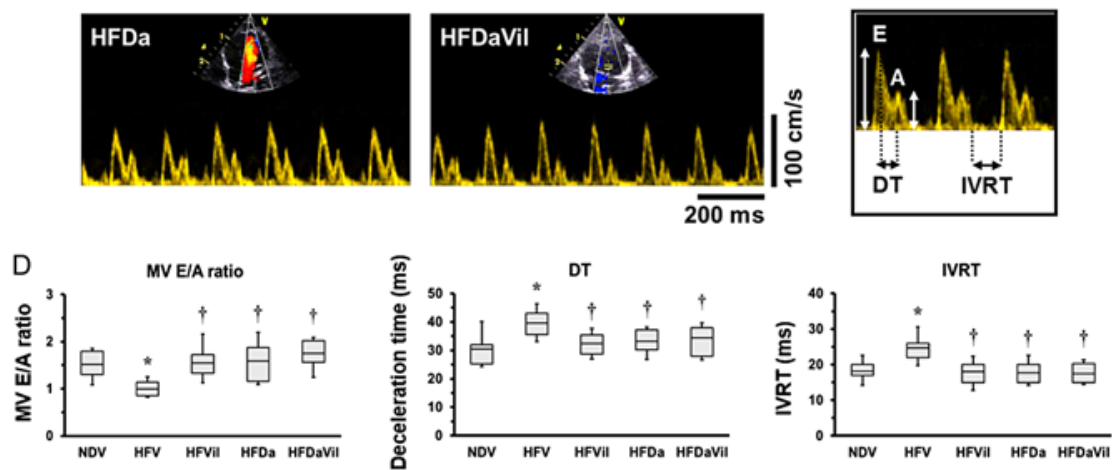

\section{Figure 2}

The effects of vildagliptin, dapagliflozin and combined therapy on systolic function, diastolic function and echocardiographic data in pre-diabetic rats. (A) M-mode of echocardiography in parasternal short axis view, $n=11-12$ per group; (B) left ventricular (LV) systolic function and echocardiographic data, $n=11-12$ per group; (C) mitral inflow by pulsed-wave Doppler in apical four-chambers view, $n=11-12$ per group and (D) LV diastolic function parameters, $n=11-12$ per group. ${ }^{*} P<0.05$ vs NDV; ${ }^{\dagger} P<0.05$ vs HFV; and ${ }^{*} P<0.05$ vs HFVil. DT, deceleration time; HFDa, high-fat diet treated with dapagliflozin; HFDaVil, high-fat diet treated with vildagliptin and dapagliflozin; HFV, high-fat diet treated with vehicle; HFVil, high-fat diet treated with vildagliptin; IVRT, isovolumetric relaxation time; IVSd, interventricular septal thickness in diastole; IVSs, interventricular septal thickness in systole; LVEF, LV ejection fraction; LVIDd, LV internal diameter in diastole; LVIDs, LV internal diameter in systole; LVPWd, LV posterior wall thickness in diastole; LVPWs, LV posterior wall thickness in systole; NDV, normal diet treated with vehicle. http://joe.endocrinology-journals.org https://doi.org/10.1530/JOE-17-0457
(C) 2018 Society for Endocrinology Published by Bioscientifica Ltd. Printed in Great Britain 
Table 2 The effects of vildagliptin, dapagliflozin and combined therapy on heart rate variability (HRV) of HFD rats after treatment for 4 weeks prior myocardial I/R injury.

\begin{tabular}{|c|c|c|c|c|c|}
\hline \multirow[b]{2}{*}{ Parameters } & \multicolumn{5}{|c|}{ Groups } \\
\hline & NDV & HFV & HFVil & HFDa & HFDaVil \\
\hline Mean HR (bpm) & $408 \pm 18$ & $497 \pm 18^{*}$ & $459 \pm 11 *,+$ & $417 \pm 17^{\dagger, \ddagger}$ & $413 \pm 17^{\dagger, \ddagger}$ \\
\hline SDNN (ms) & $4.1 \pm 0.5$ & $3.9 \pm 0.5$ & $3.9 \pm 0.5$ & $4.4 \pm 0.6$ & $4.0 \pm 0.7$ \\
\hline rMSSD (ms) & $2.1 \pm 0.1$ & $2.2 \pm 0.1$ & $2.3 \pm 0.2$ & $2.2 \pm 0.2$ & $2.1 \pm 0.1$ \\
\hline Total power (TP; ms²) & $420 \pm 45$ & $370 \pm 58$ & $490 \pm 72$ & $409 \pm 87$ & $476 \pm 69$ \\
\hline $\operatorname{VLF}\left(\mathrm{ms}^{2}\right)$ & $59 \pm 7$ & $64 \pm 6$ & $63 \pm 6$ & $63 \pm 7$ & $65 \pm 7$ \\
\hline $\mathrm{LF}\left(\mathrm{ms}^{2}\right)$ & $61 \pm 5$ & $71 \pm 8$ & $61 \pm 13$ & $50 \pm 8$ & $80 \pm 13$ \\
\hline $\mathrm{HF}\left(\mathrm{ms}^{2}\right)$ & $322 \pm 37$ & $300 \pm 23$ & $358 \pm 47$ & $308 \pm 72$ & $357 \pm 45$ \\
\hline LFnu & $0.13 \pm 0.02$ & $0.28 \pm 0.04 *$ & $0.23 \pm 0.02 *, \dagger$ & $0.16 \pm 0.02^{\dagger, \neq}$ & $0.16 \pm 0.01^{\dagger, \ddagger}$ \\
\hline HFnu & $0.85 \pm 0.03$ & $0.57 \pm 0.06 *$ & $0.72 \pm 0.05 *, \dagger$ & $0.87 \pm 0.02^{\dagger, \neq}$ & $0.88 \pm 0.02^{\dagger, \neq}$ \\
\hline LF/HF ratio & $0.21 \pm 0.03$ & $0.48 \pm 0.03 *$ & $0.34 \pm 0.06 *, \dagger$ & $0.23 \pm 0.02^{t, \neq}$ & $0.24 \pm 0.02^{\dagger, \ddagger}$ \\
\hline
\end{tabular}

Data are shown as mean \pm S.E.M., $n=10-12$ /group.

${ }^{*} P<0.05$ vs NDV; ${ }^{+} P<0.05$ vs HFV; ${ }^{\ddagger} P<0.05$ vs HFVil.

HF, high frequency; HFDa, high-fat diet treated with dapagliflozin; HFDaVil, high-fat diet treated with dapagliflozin and vildagliptin; HFnu, normalized HF (HF power to total power); HFV, high-fat diet treated with vehicle; HFVil, high-fat diet treated with vildagliptin; HR, heart rate; LF, low frequency; LFnu, normalized LF (LF power to total power); LF/HF, low frequency/high frequency; NDV, normal diet treated with vehicle; rMSSD, square root of the mean squared differences of successive NN-intervals; SDNN, standard deviation of all normal to normal intervals; TP, total power; VLF, very low frequency.

and AUCg. In addition, dapaglipflozin-treated rats had significantly increased plasma FGF21 levels, urine glucose excretion (UGE), 24-h urine volume and 24-h UGE, when compared with NDV, HFV and HFVil rats
(Supplementary Table 1). However, all pharmacological interventions demonstrated a similar efficacy on the increase of HDL-C levels and the decrease of TC and LDL-C levels, when compared with the HFV group.
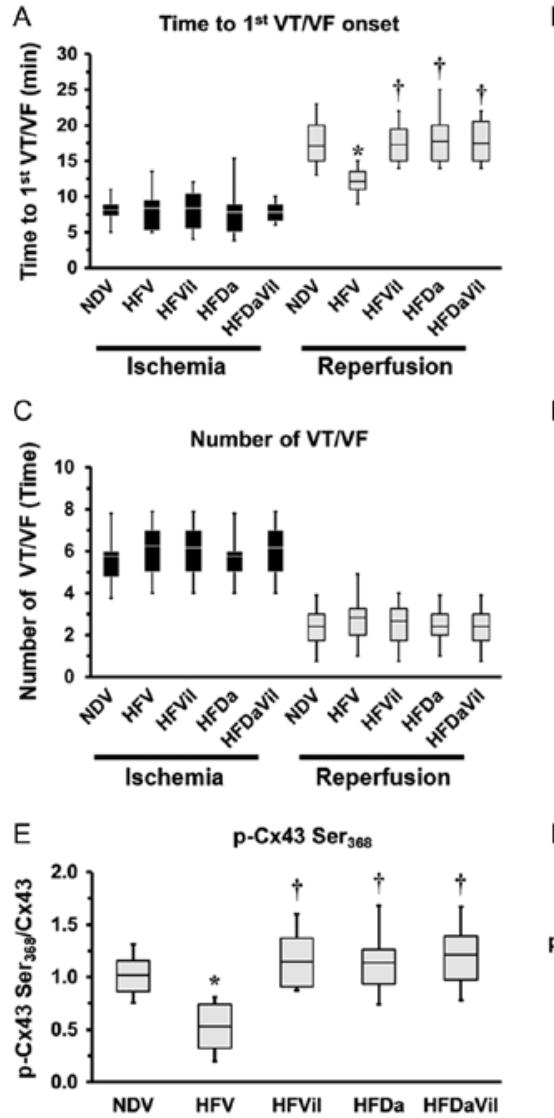

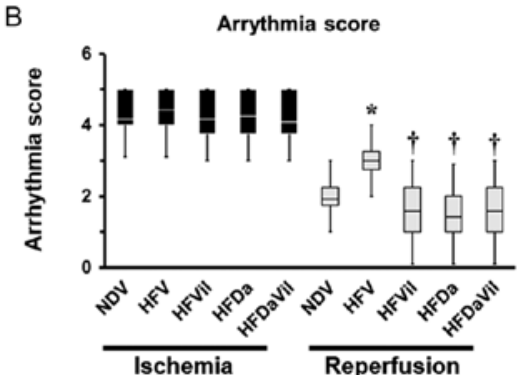

$\mathrm{D}$

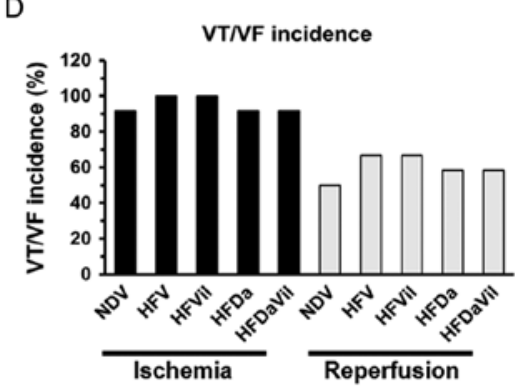

$\mathrm{F}$

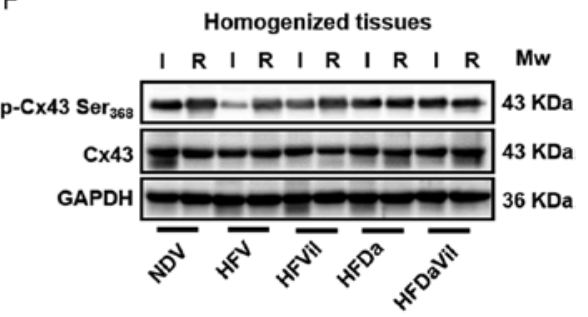

Figure 3

The effects of vildagliptin, dapagliflozin and combined therapy on cardiac arrhythmia and cardiac conductivity-related proteins in prediabetic rats with cardiac I/R injury. (A) Time to 1st VT/NF, $n=12$ per group; (B) arrhythmia score, $n=12$ per group; (C) number of VT/VF, $n=12$ per group; (D) VT/VF incidence, $n=12$ per group; (E) $\mathrm{p}-\mathrm{C} \times 43 \mathrm{Ser}_{368}$ expression in the ischemic area normalized with that in the remote area, $n=5-6$ per group and (F) representative Western blot bands of $\mathrm{p}-\mathrm{C} \times 43 \mathrm{Ser}_{368}$ and $\mathrm{C} \times 43$ proteins expression in the ischemic area and the remote area. ${ }^{*} P<0.05$ vs NDV and ${ }^{+} P<0.05$ vs HFV. Cx43, connexin 43; GAPDH, glyceraldehyde 3-phosphate dehydrogenase; HFDa, high-fat diet treated with dapagliflozin; HFDaVil, high-fat diet treated with vildagliptin and dapagliflozin; HFV, high-fat diet treated with vehicle; HFVil, high-fat diet treated with vildagliptin; NDV, normal diet treated with vehicle; $\mathrm{p}-\mathrm{C} \times 43 \mathrm{Ser}_{638}$, phosphorylation of $\mathrm{C} \times 43$ at serine638; VF, ventricular fibrillation; VT, ventricular tachycardia. 


\section{Journal of Endocrinology \\ P Tanajak et al. \\ Dapagliflozin, but not vildagliptin, led to restoration of normal blood pressure in pre-diabetic rats}

Dapagliflozin and vildagliptin in

the heart
$236: 2$

75
Blood pressure data are shown in Table 1. HFV rats had significantly increased systolic blood pressure (SBP) and mean arterial pressure (MAP), when compared with the NDV group. Dapagliflozin and combined therapy led to significantly reduced SBP and MAP, when compared with the HFV group, and the levels being restored to withinnormal limits. However, this blood pressure-lowering effect was not observed in vildagliptin-treated rats.

\section{Dapagliflozin exhibited a greater efficacy on LV function and HRV improvements than vildagliptin in pre-diabetic rats}

LV systolic and diastolic functions were evaluated, and these data are shown in Fig. 2. The representative echocardiographic images in M-mode at 4 weeks after treatment are shown in Fig. 2A. HFV rats had a decreased \%LVEF and had increased levels of LVIDs, LVPWs and LVPWd, when compared with NDV rats (Fig. 2B). The representative echocardiographic images
A

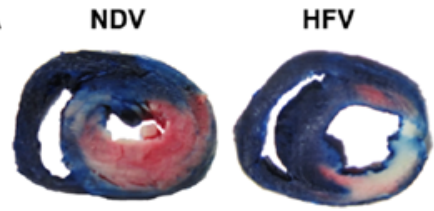

B
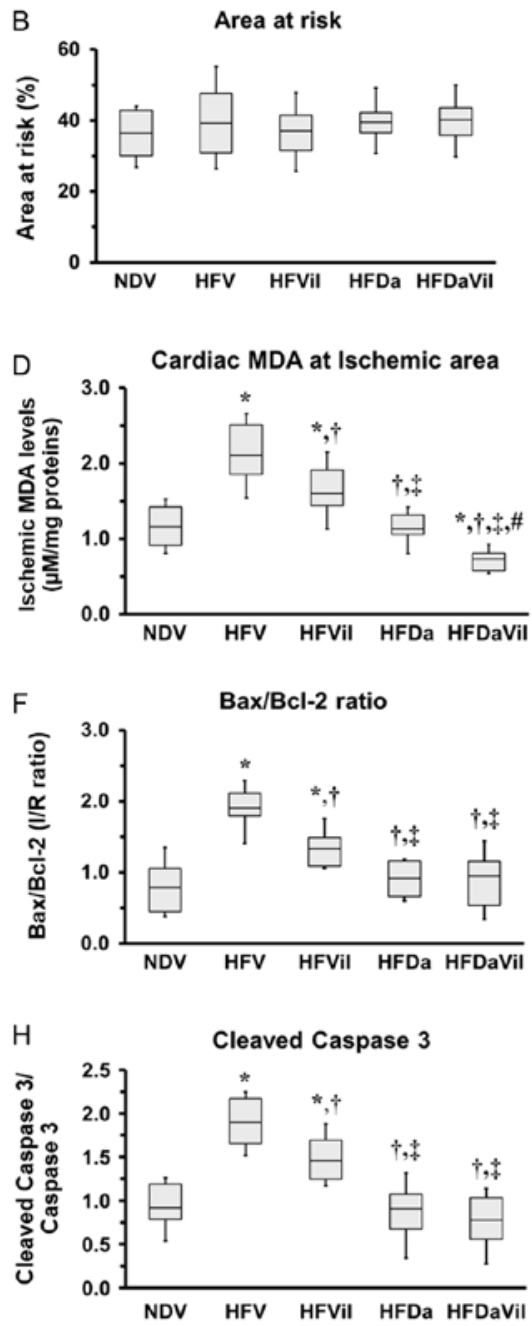

HFDa
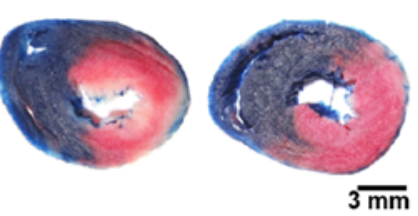

C

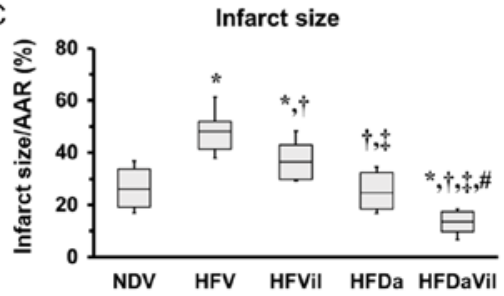

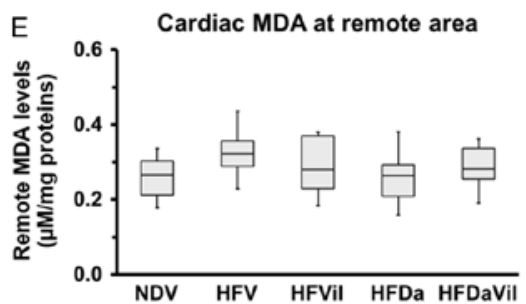

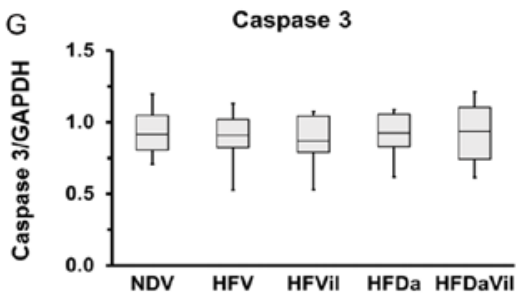

I

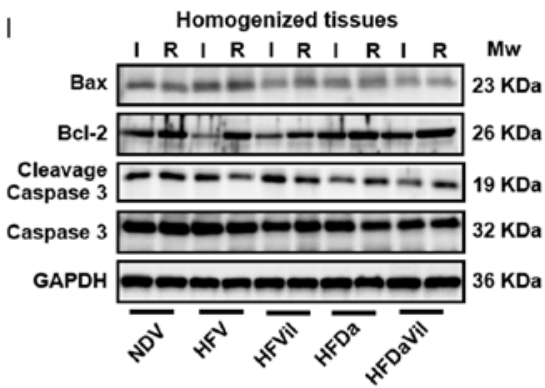

Figure 4

The effects of vildagliptin, dapagliflozin and combined therapy on myocardial infarction, myocardial lipid peroxidation and cardiac apoptotic protein expression in pre-diabetic rats with cardiac I/R injury. (A) Representative heart section for myocardial infarction, $n=6$ per group; (B) area at risk, $n=6$ per group; (C) myocardial infarct size, $n=6$ per group; (D) cardiac MDA at ischemic area, $n=5-6$ per group; (E) cardiac MDA at remote area, $n=5-6$ per group; (F) Bax/Bcl-2 ratio expression in the ischemic area normalized with that in the remote area, $n=5-6$ per group; (G) caspase 3 in the ischemic area normalized with that in the remote area, $n=6$ per group; $(\mathrm{H})$ cleaved caspase 3 in the ischemic area normalized with that in the remote area, $n=6$ per group; and (I) representative Western blot bands of Bax, Bcl-2, cleaved caspase 3 and caspase 3 proteins expression in the ischemic area and the remote area. ${ }^{*} P<0.05$ vs NDV; ${ }^{+} P<0.05$ vs HFV; ${ }^{\ddagger} P<0.05$ vs HFVil; and ${ }^{\#} P<0.05$ vs HFDa. Bax, $\mathrm{BCl}$-associared $X$ protein; $\mathrm{Bcl}$-2, B-cell lymphoma 2; GAPDH, glyceraldehyde 3-phosphate dehydrogenase; HFDa, high-fat diet treated with dapagliflozin; HFDaVil, high-fat diet treated with vildagliptin and dapagliflozin; HFV, high-fat diet treated with vehicle; HFVil, high-fat diet treated with vildagliptin; MDA, malondialdehyde; NDV, normal diet treated with vehicle. 
Table 3 The effects of vildagliptin, dapagliflozin and combined therapy on cardiac function during the myocardial I/R injury protocol.

\begin{tabular}{|c|c|c|c|}
\hline \multirow[b]{2}{*}{ Parameters } & \multicolumn{3}{|c|}{ NDV } \\
\hline & Baseline & Ischemia & Reperfusion \\
\hline HR (bpm) & $226 \pm 16$ & $338 \pm 22 *$ & $355 \pm 26 *$ \\
\hline $\mathrm{ESP}(\mathrm{mmHg})$ & $123 \pm 4$ & $77 \pm 5 *$ & $95 \pm 6 *,+$ \\
\hline EDP (mmHg) & $4 \pm 1$ & $11 \pm 3 *$ & $5 \pm 2^{\dagger}$ \\
\hline$P_{\max }(\mathrm{mmHg})$ & $136 \pm 8$ & $122 \pm 17$ & $122 \pm 9$ \\
\hline$P_{\min }(\mathrm{mmHg})$ & $5 \pm 2$ & $5 \pm 3$ & $6 \pm 2$ \\
\hline $\begin{array}{l}d P / d t_{\max } \\
\quad\left(\mathrm{mmHg} / \mathrm{s} \times 10^{3}\right)\end{array}$ & $7.81 \pm 0.41$ & $4.47 \pm 0.34 *$ & $6.22 \pm 0.50 *,+$ \\
\hline $\begin{array}{l}d P / d t_{\min } \\
\quad\left(\mathrm{mmHg} / \mathrm{s} \times 10^{3}\right)\end{array}$ & $6.51 \pm 0.38$ & $4.70 \pm 0.23 *$ & $6.01 \pm 0.20^{\dagger}$ \\
\hline $\operatorname{ESV}(\mu \mathrm{L})$ & $3 \pm 1$ & $13 \pm 2 *$ & $8 \pm 3 *,+$ \\
\hline EDV $(\mu \mathrm{L})$ & $294 \pm 18$ & $337 \pm 11 *$ & $298 \pm 31^{\dagger}$ \\
\hline $\mathrm{SV}(\mu \mathrm{L})$ & $303 \pm 21$ & $192 \pm 19 *$ & $265 \pm 11^{\dagger}$ \\
\hline $\mathrm{EF}(\%)$ & $80 \pm 2$ & $67 \pm 4 *$ & $63 \pm 4^{\dagger}$ \\
\hline $\begin{array}{l}\text { Stroke work } \\
\qquad\left(\mathrm{mmHg} \times \mu \mathrm{L} \times 10^{3}\right)\end{array}$ & $51.8 \pm 2.8$ & $33.0 \pm 1.8 *$ & $46.6 \pm 3.1^{\dagger}$ \\
\hline
\end{tabular}

\begin{tabular}{|c|c|c|}
\hline \multicolumn{3}{|c|}{ HFV } \\
\hline Baseline & Ischemia & Reperfusion \\
\hline $316 \pm 12^{\ddagger}$ & $417 \pm 31 *$ & $399 \pm 22 *$ \\
\hline $94 \pm 8^{\ddagger}$ & $56 \pm 5 *$ & $65 \pm 5 *$ \\
\hline $9 \pm 2^{\ddagger}$ & $15 \pm 4$ * & $14 \pm 3$ * \\
\hline $134 \pm 7$ & $131 \pm 17$ & $136 \pm 15$ \\
\hline $6 \pm 3$ & $6 \pm 2$ & $7 \pm 2$ \\
\hline $5.05 \pm 0.31^{\ddagger}$ & $3.72 \pm 0.3^{*}$ & $4.05 \pm 0.49 *$ \\
\hline $5.04 \pm 0.26^{\ddagger}$ & $4.15 \pm 0.24 *$ & $3.96 \pm 0.26$ * \\
\hline $9 \pm 2^{\ddagger}$ & $20 \pm 4^{*}$ & $19 \pm 4 *$ \\
\hline $329 \pm 12^{\ddagger}$ & $368 \pm 28 *$ & $375 \pm 16 *$ \\
\hline $238 \pm 18^{\ddagger}$ & $154 \pm 10$ * & $169 \pm 24$ * \\
\hline $64 \pm 3^{\ddagger}$ & $52 \pm 3 *$ & $49 \pm 3 *$ \\
\hline $38.9 \pm 3.1^{\ddagger}$ & $28.8 \pm 1.5 *$ & $29.5 \pm 2.6 *$ \\
\hline
\end{tabular}

in pulsed-wave Doppler mode demonstrating the cardiac performance, at 4 weeks after treatment, are shown in Fig. 2C. Moreover, decreased early/atrial (late) (E/A) ratio, increased deceleration time (DT) and increased isovolumetric ventricular relaxation time (IVRT) were observed in HFV rats, compared with NDV rats (Fig. 2D).

HFVil rats had significantly increased \%LVEF and decreased LVIDs, when compared with HFV rats (Fig. 2B), whereas HFDa and HFDaVil rats had increased \%LVEF with decreased LVIDs, LVPWs and LVPWd, when compared with HFV and HFVil rats (Fig. 2B). All therapeutic groups showed a similar outcome in cardiac diastolic improvement, identified by an increased E/A ratio, decreased DT and decreased IVRT, when compared with HFV rats (Fig. 2D).

HFV rats had a significantly increased mean $\mathrm{HR}$, normalized LF (LF power to total power; LFnu), LF/HF ratio and decreased normalized HF (HF power to total power; HFnu), when compared with NDV rats (Table 2). Treatment with vildagliptin significantly decreased mean HR, LFnu, LF/HF ratio and increased HFnu, when compared with HFV rats. However, dapagliflozin and the combined therapy had a greater efficacy than vildagliptin in leading to a decreased mean HR, LFnu, LF/HF ratio and increased HFnu, when compared with HFV and HFVil rats (Table 2).
Vildagliptin, dapafliflozin and combined therapy shared a similar efficacy on preventing cardiac arrhythmia in pre-diabetic rats with cardiac I/R injury

Under cardiac I/R, our results demonstrated that the prediabetic rats (HFV group) had increased susceptibility to arrhythmia during the reperfusion period, as indicated by decreased time to 1st VT/VF onset (Fig. 3A) and increased arrhythmia score (Fig. 3B), when compared with the NDV group. However, the incidence of VT/VF (Fig. 3C) and VT/VF (Fig. 3D) was not significantly altered between groups in both ischemic and reperfusion periods. The $\mathrm{HFV}$ rats also showed a significant reduction in $\mathrm{p}-\mathrm{Cx} 43$ $\mathrm{S}_{368}$ expression (Fig. 3E).

All interventions showed a similar efficacy in increased time to 1st VT/VF onset (Fig. 3A), decreased arrhythmia score (Fig. 3B) and increased p-Cx43 $\mathrm{S}_{368}$ expression (Fig. 3E) in the reperfusion period, when compared with the HFV group.

\section{Combined therapy led to a more effective outcome on myocardial infarct size reduction and LV function preservation in pre-diabetic rats with cardiac I/R injury than single regimens}

The infarct size, cardiac MDA and apoptotic protein expressions are shown in Fig. 4. HFV rats had a significantly increased myocardial infarct size (Fig. 4A). However, the 


\begin{tabular}{|c|c|c|}
\hline \multicolumn{3}{|c|}{ HFVil } \\
\hline Baseline & Ischemia & Reperfusion \\
\hline $227 \pm 18^{\#}$ & $386 \pm 28 *$ & $342 \pm 25^{*}$ \\
\hline $106 \pm 3^{\ddagger, \#}$ & $67 \pm 5^{\star}$ & $96 \pm 2^{\dagger}$ \\
\hline $7 \pm 2^{\ddagger, \#}$ & $13 \pm 3 *$ & $10 \pm 2^{+}$ \\
\hline $143 \pm 12$ & $127 \pm 16$ & $130 \pm 11$ \\
\hline $7 \pm 2$ & $7 \pm 3$ & $6 \pm 2$ \\
\hline $6.29 \pm 0.28^{\ddagger, \#}$ & $4.49 \pm 0.71 *$ & $7.16 \pm 0.57^{\dagger}$ \\
\hline $6.14 \pm 0.31^{\#}$ & $4.96 \pm 0.22 *$ & $6.23 \pm 0.28^{\dagger}$ \\
\hline $8 \pm 3^{\ddagger}$ & $14 \pm 4^{*}$ & $9 \pm 2^{+}$ \\
\hline $305 \pm 26^{\ddagger, \#}$ & $331 \pm 12 *$ & $310 \pm 16^{\dagger}$ \\
\hline $300 \pm 21^{\#}$ & $157 \pm 12 *$ & $270 \pm 17^{\dagger}$ \\
\hline $73 \pm 1^{\neq, \#}$ & $65 \pm 2 *$ & $75 \pm 1^{+}$ \\
\hline $40.8 \pm 2.5^{\ddagger}$ & $29.5 \pm 1.8^{*}$ & $42.6 \pm 1.7^{\dagger}$ \\
\hline
\end{tabular}

\begin{tabular}{c}
\hline Baseline \\
\hline $212 \pm 20^{\#}$ \\
$119 \pm 4^{\#, \$}$ \\
$5 \pm 2^{\#, \$}$ \\
$136 \pm 11$ \\
$7 \pm 4$ \\
$8.07 \pm 0.48^{\#, \$}$ \\
$6.05 \pm 0.43^{\#, \$}$ \\
$5 \pm 1 \#, \$$ \\
$280 \pm 12^{\#, \$}$ \\
$317 \pm 24^{\#}$ \\
$82 \pm 2^{\#, \$}$ \\
$50.9 \pm 3.8^{\#, \$}$ \\
\hline
\end{tabular}

\section{HFDa}

Ischemia

$333 \pm 30$ *

$86 \pm 6^{*}$

$12 \pm 2 *$

$117 \pm 13$

$6 \pm 2$

$5.32 \pm 0.37$ *

$4.85 \pm 0.29$ *

$10 \pm 4$ *

$320 \pm 24 *$

$239 \pm 14 *$

$73 \pm 2 *$

$42.0 \pm 3.0$ *

Reperfusion
$214 \pm 20^{\dagger}$
$109 \pm 5^{\dagger}$
$6 \pm 1^{\dagger}$
$127 \pm 8$
$7 \pm 3$
$7.19 \pm 0.39^{+}$

$6.11 \pm 0.39^{\dagger}$

$6 \pm 2^{\dagger}$

$2964 \pm 35^{\dagger}$

$299 \pm 18^{+}$

$79 \pm 2^{+}$

$45.3 \pm 2.6^{+}$

\begin{tabular}{|c|c|c|}
\hline \multicolumn{3}{|c|}{ HFDaVil } \\
\hline Baseline & Ischemia & Reperfusion \\
\hline $212 \pm 14^{\#}$ & $334 \pm 26 *$ & $242 \pm 16^{\dagger}$ \\
\hline $120 \pm 5^{\#, \$}$ & $108 \pm 9$ & $112 \pm 6$ \\
\hline $5 \pm 1 \#, \$$ & $7 \pm 3$ & $6 \pm 1$ \\
\hline $132 \pm 12$ & $119 \pm 9$ & $127 \pm 8$ \\
\hline $6 \pm 3$ & $6 \pm 2$ & $6 \pm 1$ \\
\hline $7.97 \pm 0.74^{\#, \$}$ & $7.72 \pm 0.82$ & $7.94 \pm 0.71$ \\
\hline
\end{tabular}

Data are shown as mean \pm S.E.M., $n=5$-6/group.

${ }^{*} P<0.05$ vs baseline; ${ }^{+} P<0.05$ vs ischemia; ${ }^{\ddagger} P<0.05$ vs baseline of NDV; $\# P<0.05$ vs baseline of $\mathrm{HFV} ; \$ P<0.05$ vs baseline of HFVil.

$d P / d t_{\max }$ maximum $d P / d t ; d P / d t_{\text {min, }}$ minimum $d P / d t$; EDP, end-diastolic pressure; EDV, end-diastolic volume; EF, ejection fraction; ESP, end-systolic pressure; ESV, end-systolic volume; HFD, high-fat diet; HFDa, high-fat diet treated with dapagliflozin; HFDaVil, high-fat diet treated with dapagliflozin and vildagliptin; HFV, high-fat diet treated with vehicle; HFVil, high-fat diet treated with vildagliptin; HR, heart rate; NDV, normal diet treated with vehicle; $P_{\max }$ maximum pressure; $P_{\min }$ minimum pressure; SV, stroke volume; SW, stroke work.

percentage of area at risk was not different between groups (Fig. 4B). HFV rats had significantly increased cardiac MDA in the ischemic areas (Fig. 4D), with no changes in cardiac MDA at remote areas (Fig. 4E), increased Bax/Bcl-2 ratio (Fig. 4F), without any changes of caspase 3 (Fig. 4G) and increased cleaved caspase 3 (Fig. 4H), when compared with the NDV group.

Although vildagliptin therapy showed a significant reduction in myocardial infarct size (Fig. 4C), cardiac MDA in the ischemic area (Fig. 4D), Bax/Bcl-2 ratio (Fig. 4F) and cleavage caspase 3 (Fig. 4H), dapagliflozin-treated rats had a greater reduction in these parameters than those on vildagliptin therapy. However, the combined therapy showed the greatest efficacy on improving myocardial infarct size (Fig. 4C) and cardiac MDA in the ischemic area (Fig. 4D) in comparison with the NDV, HFV, HFVil and HFDa groups.

The LV function parameters recorded from P-V loops during cardiac I/R are shown in Table 3. At baseline, HFV rats showed a significant reduction in LV function, indicated by reduced end-systolic pressure (ESP), $d p / d t_{\max }$, $d p / d t_{\min }, \mathrm{SV}$, ejection fraction (\%EF) and SW and also increased heart rate (HR), end-diastolic pressure (EDP), end-systolic volume (ESV) and end-diastolic volume (EDV) in comparison with the baseline in NDV rats. HFVil group had an increase in ESP, $d p / d t_{\max }, d p / d t_{\min }$, SV, \%EF and SW and a decrease in HR, EDP, ESV and $\mathrm{EDV}$, compared with the baseline measurements of the HFV rats. Dapagliflozin treatment and combined therapy showed a similar improvement indicated by the restoration of ESP, EDP, $d p / d t_{\max }, d p / d t_{\text {min }}$, ESV, EDV, SV, \%EF and SW, when compared with HFV and HFVil groups.

During the ischemic period, NDV, HFV, HFVil and HFDa groups showed a significant increase in HR, EDP, ESV and EDV and a decrease in ESP, $d p / d t_{\max }, d p / d t_{\min }, \mathrm{SV}$, $\% \mathrm{EF}$ and SW, compared with the baseline data. However, the HFDaVil group had only significantly increased HR, when compared with baseline data. During the reperfusion period, NDV, HFV, HFVil and HFDa groups had a significant decrease in EDP, ESV and EDV and an increase in ESP, $d p / d t_{\max }, d p / d t_{\min }, \mathrm{SV}, \% \mathrm{EF}$ and SW, compared with the ischemic period. HFDa and HFDaVil groups were associated with a significant reduction of $\mathrm{HR}$ in comparison with the ischemic period.

\section{Vildagliptin, dapafliflozin and combined therapy exhibited a similar efficacy on cardiac mitochondrial function, cardiac mitochondrial biogenesis and protein expression in pre-diabetic rats with cardiac I/R injury}

The data regarding cardiac mitochondrial function are presented in Fig. 5. The results showed that HFV rats had a significant increase in mitochondrial reactive oxygen species (ROS) production (Fig. 5A) 
A

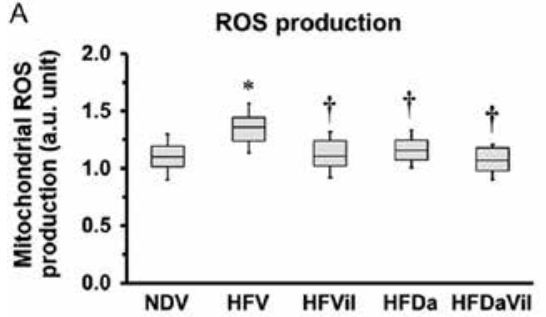

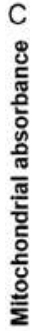

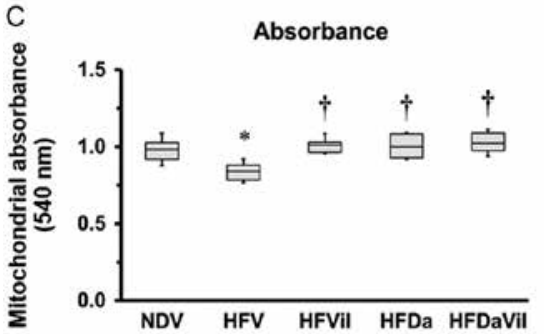

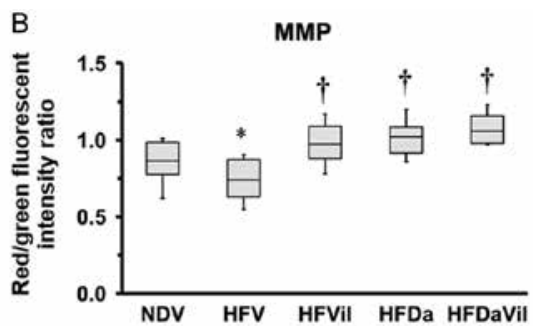

D

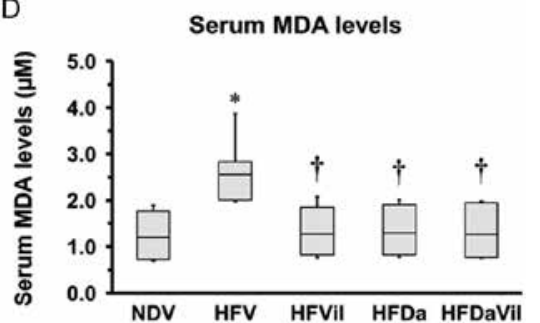

\section{Figure 5}

The effects of vildagliptin, dapagliflozin and combined therapy on cardiac mitochondrial function, cardiac mitochondrial morphology and serum lipid peroxidation in pre-diabetic rats with cardiac I/R injury. (A) Mitochondrial ROS production, $n=6$ per group; (B) mitochondrial membrane potential changes, $n=5-6$ per group; (C) mitochondrial absorbance, $n=5-6$ per group; (D) serum MDA levels, $n=5-6$ per group; and (E) representative cardiac mitochondria morphology by TEM. ${ }^{*} P<0.05$ vs NDV; ${ }^{+} P<0.05$ vs HFV; $¥ P<0.05$ vs HFVil; and $\# P<0.05$ vs HFDa. HFDa, high-fat diet treated with dapagliflozin; HFDaVil, high-fat diet treated with vildagliptin and dapagliflozin; HFV, high-fat diet treated with vehicle; HFVil, high-fat diet treated with vildagliptin; MDA, malondialdehyde; MMP, mitochondrial membrane potential; NDV, normal diet treated with vehicle; ROS, reactive oxygen species; TEM, transmission electron microscopy. and a decrease in mitochondrial red/green fluorescent intensity ratio, indicating mitochondrial depolarization (Fig. 5B), a decrease in mitochondrial absorbance intensity, indicating mitochondrial swelling (Fig. 5C) and increased serum MDA levels (Fig. 5D), compared with the NDV group. Representative electron micrographs showed impaired cardiac mitochondrial morphology in the hearts of HFV rats (Fig. 5E). This cardiac mitochondrial impairment was correlated with a significant reduction in PGC1- $\alpha$ (Fig. 6A), CPT1 (Fig. 6B) and complex I of the electron transport chain (ETC) (Fig. 6C, D, E, F and G).

All interventions had a similar efficacy in leading to improving cardiac mitochondrial function as indicated by reduced mitochondrial ROS production (Fig. 5A), increased mitochondrial red/green fluorescent intensity ratio (Fig. 5B), increased mitochondrial absorbance intensity (Fig. 5C), decreased serum MDA levels (Fig. 5D) and improved cardiac mitochondrial morphology (Fig. 5E), compared with the HFV group. These improvements were also consistent with a significant increase in PGC1- $\alpha$ (Fig. 6A), CPT1 (Fig. 6B) and complex I of the ETC (Fig. 6C).
Combined therapy exerted a greater efficacy on cardiac mitochondrial dynamics than dapagliflozin and vildagliptin in pre-diabetic rats with induced cardiac I/R injury

Cardiac mitochondrial dynamics, including mitochondrial fusion and mitochondrial fission following cardiac $I / R$ injury, are presented in Figs 7 and 8, respectively. HFV rats had decreased expression levels of cardiac mitochondrial MFN2 (Fig. 7A) and cardiac mitochondrial OPA1 (Fig. 7B). The representative bands of MFN2 and OPA1 are shown in Fig. 7C. All interventions showed a similar efficacy in increasing cardiac mitochondrial MFN2 and cardiac mitochondrial OPA1.

Regarding cardiac mitochondrial fission, there was no measurable alteration in cardiac $\mathrm{p}-\mathrm{DRP} 1 \mathrm{~S}_{616}$ between groups (Fig. 8A), but the level of cardiac p-DRP1 $\mathrm{S}_{637}$ was increased (Fig. 8B), compared with the NDV group. However, cardiac DRP1 protein expression was no difference between groups (Fig. 8C). The representative bands of cardiac p-DRP1 $\mathrm{S}_{616}, \mathrm{p}-\mathrm{DRP} 1 \mathrm{~S}_{637}$ and cardiac DRP1 protein expression are shown in Fig. 8D. HFV rats had increased expression levels of cardiac mitochondrial DRP1 (Fig. 8E), compared with the NDV group. The representative bands of cardiac 
A

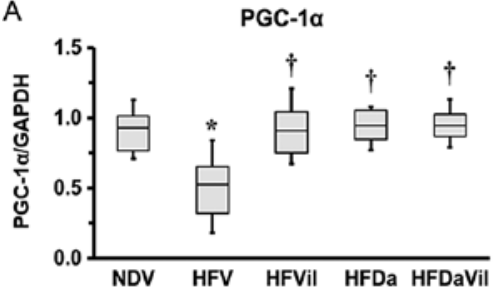

C

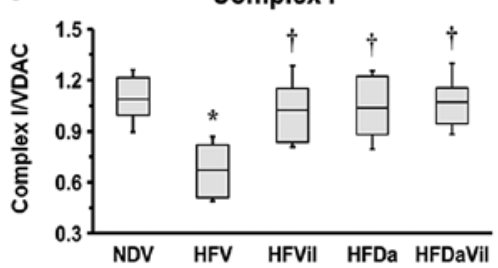

E

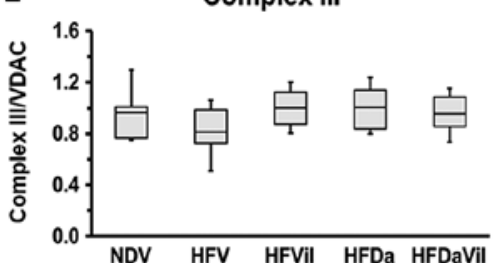

G

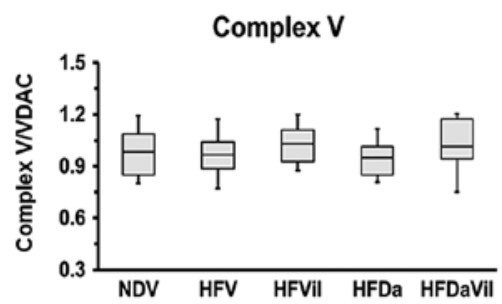

B

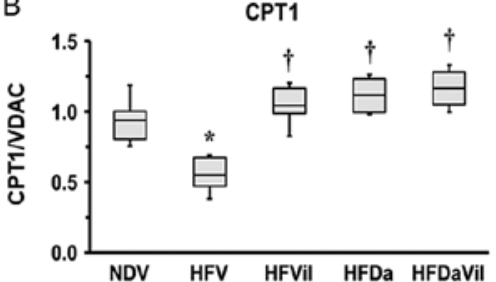

D

Complex II

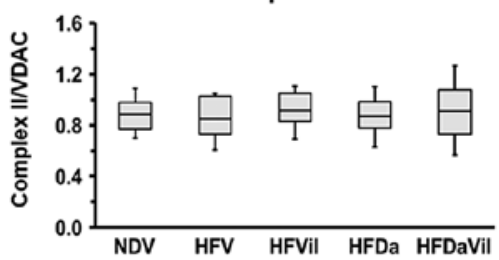

F

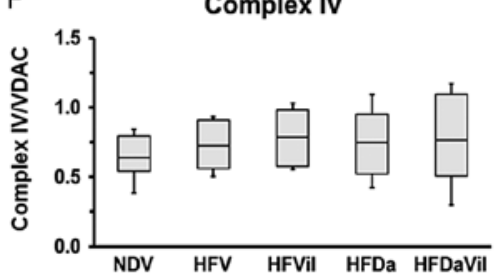

$\mathrm{H}$

Homogenized tissues

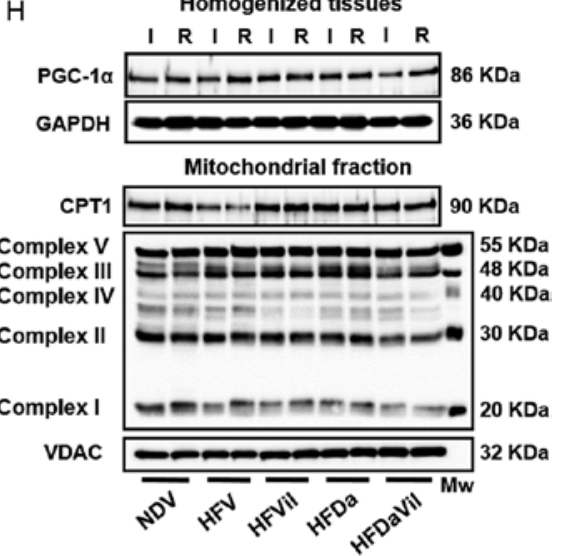

Figure 6

The effects of vildagliptin, dapagliflozin and combined therapy on proteins related to cardiac metabolism and oxidative phosphorylation in pre-diabetic rats with cardiac I/R injury. (A) Myocardial PGC1- $\alpha$ expression in the ischemic area normalized with that in the remote area, $n=6$ per group; (B) cardiac mitochondrial CPT- 1 expression in the ischemic area normalized with that in the remote area, $n=6$ per group; (C) cardiac mitochondrial complex I expression in the ischemic area normalized with that in the remote area, $n=6$ per group; (D) cardiac mitochondrial complex II expression in the ischemic area normalized with that in the remote area, $n=6$ per group; (E) cardiac mitochondrial complex III expression in the ischemic area normalized with that in the remote area, $n=6$ per group; (F) cardiac mitochondrial complex IV expression in the ischemic area normalized with that in the remote area, $n=6$ per group; (G) cardiac mitochondrial complex $V$ expression in the ischemic area normalized with that in the remote area, $n=6$ per group; and $(H)$ representative Western blot bands of at PGC1- $\alpha$, CPT1, complex I, $\mathrm{II}, \mathrm{III}, \mathrm{VI}$ and $\mathrm{V}$ in the ischemic area and the remote area. ${ }^{*} P<0.05$ vs NDV; ${ }^{+} P<0.05$ vs HFV. CPT1, carnitine palmitoyltransferase I; GAPDH, glyceraldehyde 3-phosphate dehydrogenase; HFDa, high-fat diet treated with dapagliflozin HFDaVil, high-fat diet treated with vildagliptin and dapagliflozin; HFV, high-fat diet treated with vehicle; HFVil, high-fat diet treated with vildagliptin; NDV, normal diet treated with vehicle; PGC1- $\alpha$, peroxisome proliferator-activated receptor gamma coactivator 1-alpha; VDAC, voltage-dependent anion channel. mitochondrial DRP1 protein expression are shown in Fig. 8F. All interventions showed a similar efficacy in increasing cardiac p-DRP1 $\mathrm{S}_{637}$ expression, when compared with the HFV group. Although dapagliflozin exerted a more enhanced reduction in cardiac mitochondrial DRP1 expression than vildagliptin, combined therapy showed the greatest efficacy in this reduction, when compared with the NDV, HFV, HFVil and HFDa groups (Fig. 8E).

\section{Discussion}

The major findings in this study can be summarized as follows: (1) long-term HFD consumption led to obeseinsulin resistance, increased blood pressure, decreased
HRV and impaired LV function; (2) dapagliflozin exerted greater efficacy than vildagliptin in metabolic regulation, HRV, blood pressure and LV function improvements (3) under conditions of cardiac I/R condition, obese insulinresistant rats had a reduction in the expression of proteins related to the management of cardiac metabolism, cardiac mitochondrial function, complex I of the ETC, cardiac mitochondrial fusion and an increase in the expression of proteins related to cardiac mitochondrial fission and myocyte apoptosis, leading to larger infarct size than that seen in ND rats; (4) although dapagliflozin had more beneficial effects on cardioprotection than vildagliptin in pre-diabetic rats with initiated cardiac I/R injury, a combined therapy of the two agents demonstrated greater 
A
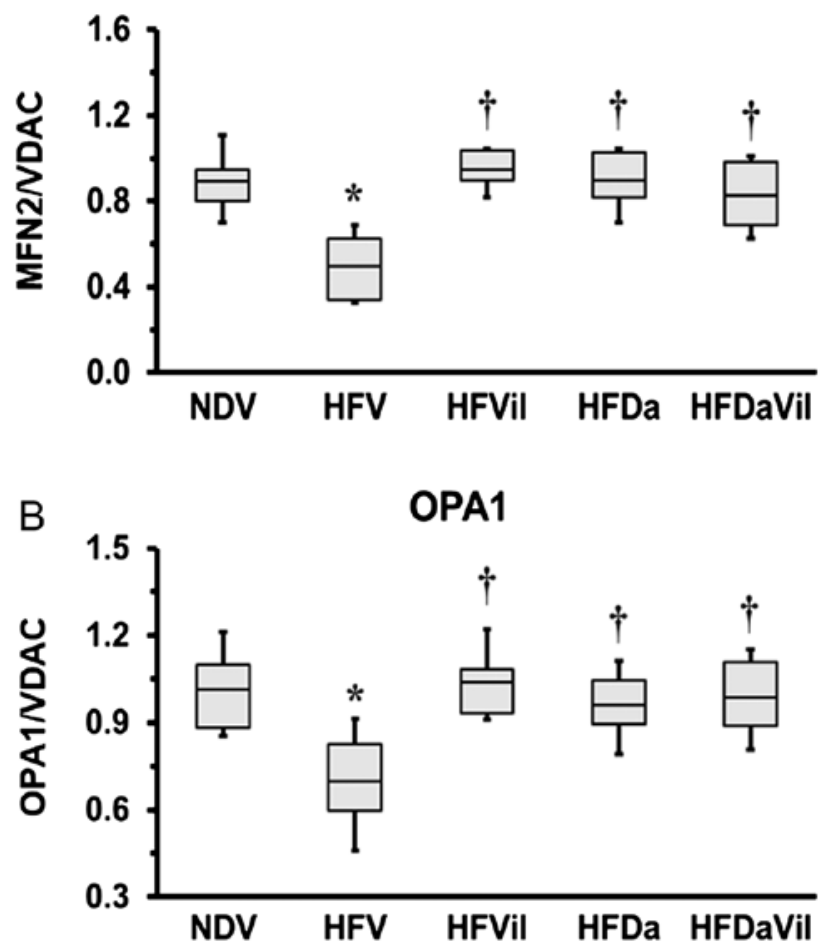

C

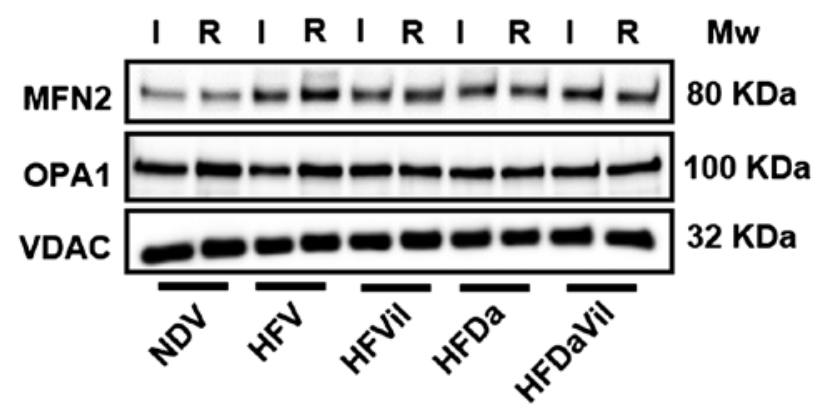

Figure 7

The effects of vildagiptin, dapagliflozin and combined therapy on cardiac mitochondrial fusion in pre-diabetic rats with cardiac I/R injury. (A) Cardiac mitochondrial MFN2 expression in the ischemic area normalized with that in the remote area, $n=5-6$ per group; (B) cardiac mitochondrial OPA1 expression in the ischemic area normalized with that in the remote area, $n=5-6$ per group; and (C) representative Western blot bands of MFN2 and OPA1 using cardiac mitochondrial fraction as a samples. ${ }^{*} P<0.05$ vs NDV; and $+P<0.05$ vs HFV. HFDa, high-fat diet treated with dapagliflozin; HFDaVil, high-fat diet treated with vildagliptin and dapagliflozin; HFV, high-fat diet treated with vehicle; HFVil, high-fat diet treated with vildagliptin; MFN2, mitofusin 2; NDV, normal diet treated with vehicle; OPA1, optic atrophy 1; VDAC, voltage-dependent anion channel.

cardioprotective effects than either of the single regimens. Evidence suggests that this outcome was due to markedly decreased mitochondrial fission and cardiac oxidative stress, leading to the largest reduction in myocardial infarct size in HFDaVil rats with cardiac I/R injury (Fig. 9).
Alhough these drugs are for the treatment of type 2 diabetes, we believe that early treatment and prevention since pre-diabetic state is crucial and have been investigating the roles of pharmacological interventions as well as device intervention in pre-diabetic conditions like the obese insulin-resistant model used in this study for many years (Pipatpiboon et al. 2013, Apaijai et al. 2014, 2016, Chinda et al. 2014, Tanajak et al. 2017). In addition, due to the fast growing number of obese insulinresistant population worldwide, we believe that early interventions in this group prior to the development into type 2 diabetes will provide impacts on therapeutic strategies as well as possible reduction in economic burden. Moreover, there are many studies investigating the effects of the drugs in type 2 diabetes, but only a few that investigates these effects under pre-diabetic obese insulin-resistant condition as well as their cardioprotective effects. Furthermore, regarding dapagliflozin, previous studies demonstrated that it not only exerts the beneficial effects on glucose lowering in diabetes models, but also has the pleiotrophic effects such as reduced body weight, reduced blood pressure, improved metabolic regulation, renoprotection and neuroprotection in non-diabetic models (Han et al. 2008, Lee et al. 2017a, Lundkvist et al. 2017, Sa-Nguanmoo et al. 2017). For these, we aimed to investigate the cardioprotective efficacy of dapagliflozin in comparison to vildagliptin and combined therapy in a pre-diabetic obese insulin-resistant model, which has never been investigated. We believe that the findings from this study could provide insights for future clinical application in obese insulin-resistant patients especially those with cardiac ischemia/reperfusion injury.

In the pre-diabetic condition, dapagliflozin showed a greater efficacy in restoring metabolic regulation, HRV and LV function when compared with vildagliptin. Also only rats treated with dagpagliflozin showed a significant reduction in SBP and mean arterial pressure, which was not found in vildagliptin-treated rats. Previous studies demonstrated that dapagliflozin therapy caused a reduction in blood pressure due to diuretic effects (Lambers Heerspink et al. 2013, Weber et al. 2016). These findings could be deemed similar to our results as our data also indicated an increase in the level of urine excretion in dapagliflozin-treated rats, which could play an important role in blood pressure reduction and weight reduction.

HRV has been identified as a powerful independent predictor of increasing mortality in post myocardial infarction patients (Kleiger et al. 1987). HRV became acknowledged as an indicator of cardiac sympathovagal imbalance in numerous clinical studies to identify patients 
A

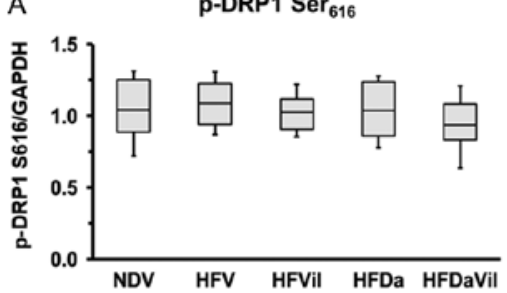

C

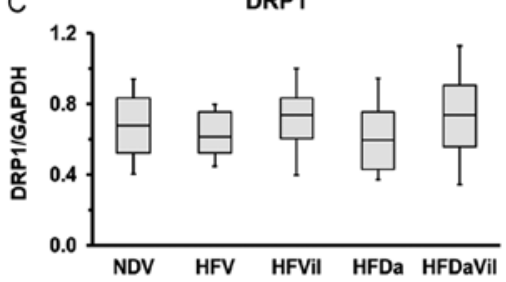

E

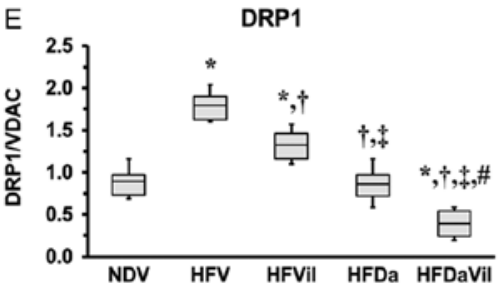

B

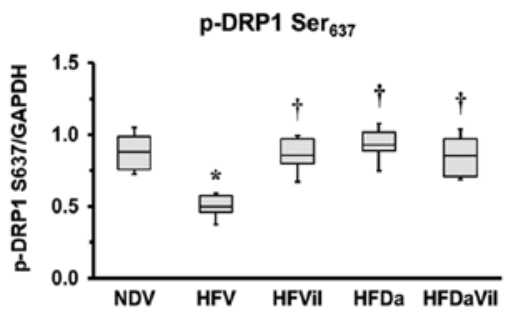

D

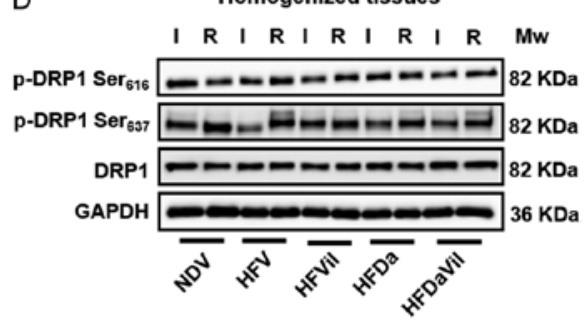

$\mathrm{F}$
Figure 8

The effects of vildagliptin, dapagliflozin and combined therapy on cardiac mitochondrial fission in pre-diabetic rats with cardiac I/R injury. (A) Myocardial p-DRP1 Ser $_{616}$ expression in the ischemic area normalized with that in the remote area, $n=5-6$ per group; (B) myocardial p-DRP1 $\mathrm{Ser}_{637}$ expression in the ischemic area normalized with that in the remote area, $n=5-6$ per group; (C) myocardial DRP1 expression in the ischemic area normalized with that in the remote area, $n=6 /$ group; (D) representative Western blot bands of p-DRP1 Ser ${ }_{616}$, p-DRP1 Ser ${ }_{637}$ using myocardial tissues as samples; (E) cardiac mitochondrial DRP1 expression in the ischemic area normalized with that in the remote area, $n=6$ per group and (F) representative Western blot bands of DRP1 using cardiac mitochondrial fraction as a samples. ${ }^{*} P<0.05$ vs NDV; ${ }^{+} P<0.05$ vs HFV; ${ }^{\ddagger} P<0.05$ vs HFVil; and $\# P<0.05$ vs HFDa. DRP1, dynamin-related protein 1; GAPDH, glyceraldehyde 3-phosphate dehydrogenase; HFDa, high-fat diet treated with dapagliflozin; HFDaVil, high-fat diet treated with vildagliptin and dapagliflozin; HFV, high-fat diet treated with vehicle; HFVil, high-fat diet treated with vildagliptin; NDV, normal diet treated with vehicle; p-DRP1 Ser $_{616}$, phosphorylation of DRP1 at serine616; p-DRP1 Ser ${ }_{637}$, phosphorylation of DRP1 at serine637; VDAC, voltage-dependent anion channel. who have an increased risk of cardiovascular mortality in populations with and without known CVD (Bigger et al. 1992, La Rovere et al. 1998, Liao et al. 2002, Hillebrand et al. 2013). Our study demonstrated that pre-diabetic rats had impaired HRV due to the shift of cardiac autonomic balance towards sympathetic dominance. In the present study, dapagliflozin exerted more effective HRV restoration than the vildagliptin treatment, suggesting that dapagliflozin therapy exerts a higher efficacy on improved cardiac autonomic control in the pre-diabetic condition than vildagliptin.

Fatty acid oxidation is a major source of ATP from cardiac energy metabolism and the alteration of cardiac metabolism can reduce cardiac efficiency (Lopaschuk et al. 2010). Cardiac mitochondrial metabolism-related proteins PGC1- $\alpha$ and CPT1 are essential proteins for the regulation of cardiac fatty acid oxidation (Duncan 2011, Lucas et al. 2016). Our results showed that PGC1- $\alpha$ and CPT1 levels were decreased in the I/R hearts of obese insulin-resistant rats. Although previous studies demonstrated the reduction in myocardial PGC1- $\alpha$ and CPT-1 protein expression in genetic obesity model (Young et al. 2002) and HFD-induced obese models (Neves et al. 2014, Tanajak et al. 2016, 2017), the alteration in cardiac PGC1- $\alpha$ protein expression and cardiac mitochondrial CPT-1 protein expression in HFD-induced obese insulin-resistant with myocardial $\mathrm{I} / \mathrm{R}$ injury model compared with lean model has never been reported. This study is the first to demonstrate these impairments. In addition, the expression of cardiac mitochondrial complex I of the ETC and cardiac mitochondrial function were reduced in these HFV rats after I/R injury. Therefore, reduced levels of cardiac PGC1- $\alpha$, cardiac mitochondrial CPT1, cardiac mitochondrial complex I of the ETC and decreased cardiac mitochondrial function, may all be responsible for the reduction in cardiac energy metabolism in cases of I/R injury. Regarding pharmacological interventions in this study, we found that vildagliptin, dapagliflozin and combined therapy, showed a similar efficacy on increasing the expression of cardiac mitochondrial metabolism-related proteins PGC1- $\alpha$ and CPT1, the cardiac mitochondrial complex I of the ETC and also improving cardiac mitochondrial function. Regarding protein-related cardiac arrhythmias Cx43 and arrythmogenesis profiles, a decreasing in gap junction protein p-Cx43 $\mathrm{S}_{368}$ expression, decreasing in time to first VT/VF onset and increasing arrhythmia score during I/R injury were found in HFV rats. All interventions gave a similar efficacy by increasing gap junction protein p-Cx43 $\mathrm{S}_{368}$ expression. This could be mainly responsible for reducing the arrhythmia vulnerability during I/R injury in these rats.

An inhibition of cardiac mitochondrial fission could result in cardioprotection against myocardial I/R injury 


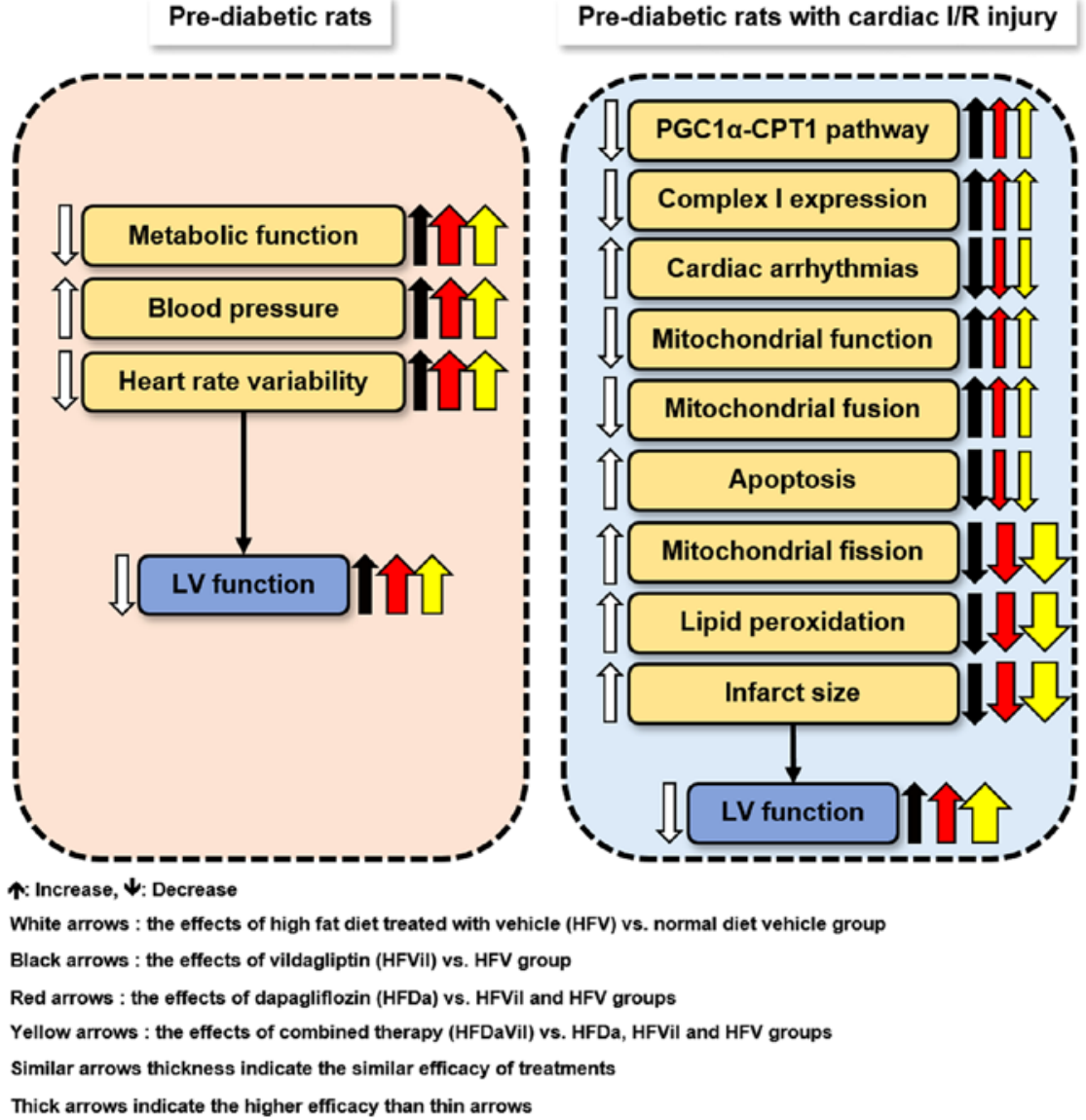

Figure 9

Diagram summarizing the effects of high-fat diet consumption, vildagliptin, dapagliflozin and combined therapy in pre-diabetic rats and pre-diabetic rats with cardiac I/R injury. CPT1, carnitine palmitoyltransferase I; I/R, ischemia/ reperfusion; LV, left ventricle; PGC-1 $\alpha$, peroxisome proliferator-activated receptor gamma coactivator 1-alpha. A full color version of this figure is available at http://dx.doi.org/10.1530/ JOE-17-0457.
(Ong et al. 2010), and this has also been seen to enhance survival in a murine cardiac arrest model (Sharp et al. 2015). Regarding the results of cardiac mitochondrial dynamics in this study, we found that cardiac mitochondrial fission was increased and mitochondrial fusion was decreased in HFV rats with I/R injury. Although all intervention arms had a similar efficacy on increasing cardiac mitochondrial fusion after cardiac I/R, dapagliflozin therapy had a higher efficacy than vildagliptin therapy, the greater impact being identified by a decrease in cardiac mitochondrial fission. However, the combined therapy exerted the greatest efficacy on reducing cardiac mitochondrial fission after cardiac $I / R$. This study is the first to demonstrate the efficacy of pharmacological intervention (vildaliptin and dapagliflozin) on cardiac mitochondrial dynamic improvements in pre-diabetic rats with cardiac I/R injury. Although dapagliflozin showed a higher efficacy in decreasing cardiac mitochondrial fission than vildagliptin, a combination therapy of the 2 agents showed the highest efficacy as regards cardiac mitochondrial fission reduction through a marked reduction in cardiac mitochondrial DRP1 expression. However, all pharmacological intervention gave a similar efficacy in cardiac mitochondrial fusion through increasing cardiac mitochondrial MFN2 and OPA1 expression. These findings indicate that combined therapy provides a better restoration of cardiac mitochondrial dynamics than either of the single regimens. In addition, the combined therapy provided the most effective outcome for cardioprotection following myocardial I/R potentially through a reduction in both mitochondrial fission and cardiac MDA levels being seen in the ischemic areas. In this study, cardiac mitophagy was not investigated as a further determinant. Since mitophagy plays an important role in controlling the mitochondrial fragmentation (Burman et al. 2017, Meyer et al. 2017) and also potentially the determinant of the cardiac function in this case (Li et al. 2016, Feng et al. 2017, Lee et al. 2017b, Zhou et al. 2017), future studies are needed to confirm the findings in the present study.

In conclusion, dapagliflozin exerted a greater efficacy on the regulation of metabolism, the balance of the cardiac autonomic system, reduction of blood pressure and improvement in LV function, compared to vildagliptin in pre-diabetic rats. However, under conditions of cardiac I/R, although dapagliflozin had greater efficacy than vildagliptin, combined therapy provided the 
more enhanced cardioprotective benefits by reducing myocardial infarct size and improving LV function. The outstanding efficacy of the combined therapy potentially through a reduction in mitochondrial fission and cardiac MDA levels.

\section{Supplementary data}

This is linked to the online version of the paper at https://doi.org/10.1530/ JOE-17-0457.

\section{Declaration of interest}

The authors declare that there is no conflict of interest that could be perceived as prejudicing the impartiality of research reported.

\section{Funding}

This work was supported by a NSTDA Research Chair Grant from the National Science and Technology Development Agency Thailand (N C); the Thailand Research Fund RTA6080003 (S C) and the Royal Golden Jubilee Ph.D. Program (P T and S C C) and Chiang Mai University Center of Excellence Award (N C).

\section{Author contribution statement}

Conception and design of experiments: $S \mathrm{C}$ and $\mathrm{N} \mathrm{C}$. Conduction of experiments: P T, P S, S S and S T. Data analysis: P T, P S, S S, S T, N S, S C and N C. Manuscript construction: P T, S C and N C.

\section{Acknowledgements}

The authors would like to thank Maria Love for her editorial assistance.

\section{References}

Apaijai N, Pintana H, Chattipakorn SC \& Chattipakorn N 2013 Effects of vildagliptin versus sitagliptin, on cardiac function, heart rate variability and mitochondrial function in obese insulin-resistant rats. British Journal of Pharmacology 169 1048-1057. (https://doi. org/10.1111/bph.12176)

Apaijai N, Chinda K, Palee S, Chattipakorn S \& Chattipakorn N 2014 Combined vildagliptin and metformin exert better cardioprotection than monotherapy against ischemia-reperfusion injury in obeseinsulin resistant rats. PLOS ONE 9 e102374. (https://doi.org/10.1371/ journal.pone.0102374)

Apaijai N, Inthachai T, Lekawanvijit S, Chattipakorn SC \& Chattipakorn N 2016 Effects of dipeptidyl peptidase-4 inhibitor in insulin-resistant rats with myocardial infarction. Journal of Endocrinology 229 245-258. (https://doi.org/10.1530/JOE-16-0096)

Bigger JT Jr, Fleiss JL, Steinman RC, Rolnitzky LM, Kleiger RE \& Rottman JN 1992 Frequency domain measures of heart period variability and mortality after myocardial infarction. Circulation $\mathbf{8 5}$ 164-171. (https://doi.org/10.1161/01.CIR.85.1.164)
Burman JL, Pickles S, Wang C, Sekine S, Vargas JNS, Zhang Z, Youle AM, Nezich CL, Wu X, Hammer JA, et al. 2017 Mitochondrial fission facilitates the selective mitophagy of protein aggregates. Journal of Cell Biology 216 3231-3247. (https://doi.org/10.1083/jcb.201612106)

Chinda K, Sanit J, Chattipakorn S \& Chattipakorn N 2014 Dipeptidyl peptidase- 4 inhibitor reduces infarct size and preserves cardiac function via mitochondrial protection in ischaemia-reperfusion rat heart. Diabetes and Vascular Disease Research 11 75-83. (https://doi. org/10.1177/1479164113516134)

Curtis MJ, Hancox JC, Farkas A, Wainwright CL, Stables CL, Saint DA, Clements-Jewery H, Lambiase PD, Billman GE, Janse MJ, et al. 2013 The Lambeth Conventions (II): guidelines for the study of animal and human ventricular and supraventricular arrhythmias. Pharmacology and Therapeutics 139 213-248. (https://doi.org/10.1016/j. pharmthera.2013.04.008)

Duncan JG 2011 Peroxisome proliferator activated receptor-alpha (PPARalpha) and PPAR gamma coactivator-1alpha (PGC-1alpha) regulation of cardiac metabolism in diabetes. Pediatric Cardiology $\mathbf{3 2}$ 323-328. (https://doi.org/10.1007/s00246-011-9889-8)

Feng Y, Madungwe NB, da Cruz Junho CV \& Bopassa JC 2017 Activation of $\mathrm{G}$ protein-coupled estrogen receptor 1 at the onset of reperfusion protects the myocardium against ischemia/reperfusion injury by reducing mitochondrial dysfunction and mitophagy. British Journal of Pharmacology 174 4329-4344. (https://doi.org/10.1111/bph.14033)

Han S, Hagan DL, Taylor JR, Xin L, Meng W, Biller SA, Wetterau JR, Washburn WN \& Whaley JM 2008 Dapagliflozin, a selective SGLT2 inhibitor, improves glucose homeostasis in normal and diabetic rats. Diabetes 57 1723-1729. (https://doi.org/10.2337/db07-1472)

He YL, Zhang Y, Serra D, Wang Y, Ligueros-Saylan M \& Dole WP 2011 Thorough QT study of the effects of vildagliptin, a dipeptidyl peptidase IV inhibitor, on cardiac repolarization and conduction in healthy volunteers. Current Medical Research and Opinion 27 1453-1463. (https://doi.org/10.1185/03007995.2011.585395)

Hillebrand S, Gast KB, de Mutsert R, Swenne CA, Jukema JW, Middeldorp S, Rosendaal FR \& Dekkers OM 2013 Heart rate variability and first cardiovascular event in populations without known cardiovascular disease: meta-analysis and dose-response metaregression. Europace 15 742-749. (https://doi.org/10.1093/europace/ eus341)

Kilkenny C, Browne W, Cuthill IC, Emerson M, Altman DG \& Group NCRRGW 2010 Animal research: reporting in vivo experiments: the ARRIVE guidelines. British Journal of Pharmacology 160 1577-1579. (https://doi.org/10.1111/j.1476-5381.2010.00872.x)

Kleiger RE, Miller JP, Bigger JT Jr \& Moss AJ 1987 Decreased heart rate variability and its association with increased mortality after acute myocardial infarction. American Journal of Cardiology 59 256-262. (https://doi.org/10.1016/0002-9149(87)90795-8)

La Rovere MT, Bigger JT Jr, Marcus FI, Mortara A \& Schwartz PJ 1998 Baroreflex sensitivity and heart-rate variability in prediction of total cardiac mortality after myocardial infarction. ATRAMI (Autonomic Tone and Reflexes After Myocardial Infarction) Investigators. Lancet 351 478-484. (https://doi.org/10.1016/S0140-6736(97)11144-8)

Lambers Heerspink HJ, de Zeeuw D, Wie L, Leslie B \& List J 2013 Dapagliflozin a glucose-regulating drug with diuretic properties in subjects with type 2 diabetes. Diabetes, Obesity and Metabolism 15 853-862. (https://doi.org/10.1111/dom.12127)

Lee TM, Chang NC \& Lin SZ 2017a Dapagliflozin, a selective SGLT2 Inhibitor, attenuated cardiac fibrosis by regulating the macrophage polarization via STAT3 signaling in infarcted rat hearts. Free Radical Biology and Medicine 104 298-310. (https://doi.org/10.1016/j. freeradbiomed.2017.01.035)

Lee Y, Kwon I, Jang Y, Song W, Cosio-Lima LM \& Roltsch MH $2017 b$ Potential signaling pathways of acute endurance exerciseinduced cardiac autophagy and mitophagy and its possible role in cardioprotection. Journal of Physiological Sciences 67 639-654. (https:// doi.org/10.1007/s12576-017-0555-7)
() 2018 Society for Endocrinology Published by Bioscientifica Ltd. Printed in Great Britain 
Li Q, Dong QT, Yang YJ, Tian XQ, Jin C, Huang PS, Jiang LP \& Chen GH 2016 AMPK-mediated cardioprotection of atorvastatin relates to the reduction of apoptosis and activation of autophagy in infarcted rat hearts. American Journal of Translational Research 8 4160-4171.

Liao D, Carnethon M, Evans GW, Cascio WE \& Heiss G 2002 Lower heart rate variability is associated with the development of coronary heart disease in individuals with diabetes: the atherosclerosis risk in communities (ARIC) study. Diabetes 51 3524-3531. (https://doi. org/10.2337/diabetes.51.12.3524)

Lopaschuk GD, Ussher JR, Folmes CD, Jaswal JS \& Stanley WC 2010 Myocardial fatty acid metabolism in health and disease. Physiological Reviews 90 207-258. (https://doi.org/10.1152/physrev.00015.2009)

Lucas E, Vila-Bedmar R, Arcones AC, Cruces-Sande M, Cachofeiro V, Mayor F Jr \& Murga C 2016 Obesity-induced cardiac lipid accumulation in adult mice is modulated by $\mathrm{G}$ protein-coupled receptor kinase 2 levels. Cardiovascular Diabetology 15 155. (https:// doi.org/10.1186/s12933-016-0474-6)

Lundkvist P, Sjostrom CD, Amini S, Pereira MJ, Johnsson E \& Eriksson JW 2017 Dapagliflozin once-daily and exenatide once-weekly dual therapy: a 24-week randomized, placebo-controlled, phase II study examining effects on body weight and prediabetes in obese adults without diabetes. Diabetes, Obesity and Metabolism 19 49-60. (https:// doi.org/10.1111/dom.12779)

Meyer JN, Leuthner TC \& Luz AL 2017 Mitochondrial fusion, fission, and mitochondrial toxicity. Toxicology 391 42-53. (https://doi. org/10.1016/j.tox.2017.07.019)

Neves FA, Cortez E, Bernardo AF, Mattos AB, Vieira AK, Malafaia Tde O, Thole AA, Rodrigues-Cunha AC, Garcia-Souza EP, Sichieri R, et al. 2014 Heart energy metabolism impairment in Western-diet induced obese mice. Journal of Nutritional Biochemistry 25 50-57. (https://doi. org/10.1016/j.jnutbio.2013.08.014)

Ong SB, Subrayan S, Lim SY, Yellon DM, Davidson SM \& Hausenloy DJ 2010 Inhibiting mitochondrial fission protects the heart against ischemia/reperfusion injury. Circulation 121 2012-2022. (https://doi. org/10.1161/CIRCULATIONAHA.109.906610)

Pipatpiboon N, Pintana H, Pratchayasakul W, Chattipakorn N \& Chattipakorn SC 2013 DPP4-inhibitor improves neuronal insulin receptor function, brain mitochondrial function and cognitive function in rats with insulin resistance induced by high-fat diet consumption. European Journal of Neuroscience 37 839-849. (https:// doi.org/10.1111/ejn.12088)

Pongkan W, Chattipakorn SC \& Chattipakorn N 2015 Chronic testosterone replacement exerts cardioprotection against cardiac ischemia-reperfusion injury by attenuating mitochondrial dysfunction in testosterone-deprived rats. PLOS ONE 10 e0122503. (https://doi.org/10.1371/journal.pone.0122503)

Pongkan W, Pintana H, Jaiwongkam T, Kredphoo S, Sivasinprasasn S, Chattipakorn SC \& Chattipakorn N 2016 Vildagliptin reduces cardiac ischemic-reperfusion injury in obese orchiectomized rats. Journal of Endocrinology 231 81-95. (https://doi.org/10.1530/JOE-16-0232)
Riess ML, Rhodes SS, Stowe DF, Aldakkak M \& Camara AK 2009 Comparison of cumulative planimetry versus manual dissection to assess experimental infarct size in isolated hearts. Journal of Pharmacological and Toxicological Methods 60 275-280. (https://doi. org/10.1016/j.vascn.2009.05.012)

Sa-Nguanmoo P, Tanajak P, Kerdphoo S, Jaiwongkam T, Pratchayasakul W, Chattipakorn N \& Chattipakorn SC 2017 SGLT2-inhibitor and DPP-4 inhibitor improve brain function via attenuating mitochondrial dysfunction, insulin resistance, inflammation, and apoptosis in HFDinduced obese rats. Toxicology and Applied Pharmacology 333 43-50. (https://doi.org/10.1016/j.taap.2017.08.005)

Sharp WW, Beiser DG, Fang YH, Han M, Piao L, Varughese J \& Archer SL 2015 Inhibition of the mitochondrial fission protein dynaminrelated protein 1 improves survival in a murine cardiac arrest model. Critical Care Medicine 43 e38-e47. (https://doi.org/10.1097/ CCM.0000000000000817)

Tanajak P, Sa-Nguanmoo P, Wang X, Liang G, Li X, Jiang C, Chattipakorn SC \& Chattipakorn N 2016 Fibroblast growth factor 21 (FGF21) therapy attenuates left ventricular dysfunction and metabolic disturbance by improving FGF21 sensitivity, cardiac mitochondrial redox homoeostasis and structural changes in pre-diabetic rats. Acta Physiologica 217 287-299. (https://doi.org/10.1111/apha.12698)

Tanajak P, Pintana H, Siri-Angkul N, Khamseekaew J, Apaijai N, Chattipakorn SC \& Chattipakorn N 2017 Vildagliptin and caloric restriction for cardioprotection in pre-diabetic rats. Journal of Endocrinology 232 189-204. (https://doi.org/10.1530/JOE-16-0406)

Thummasorn S, Kumfu S, Chattipakorn S \& Chattipakorn N 2011 Granulocyte-colony stimulating factor attenuates mitochondrial dysfunction induced by oxidative stress in cardiac mitochondria. Mitochondrion 11 457-466. (https://doi.org/10.1016/j.mito.2011.01.008)

Vrhovac I, Balen Eror D, Klessen D, Burger C, Breljak D, Kraus O, Radovic N, Jadrijevic S, Aleksic I, Walles T, et al. 2015 Localizations of $\mathrm{Na}(+)$-D-glucose cotransporters SGLT1 and SGLT2 in human kidney and of SGLT1 in human small intestine, liver, lung, and heart. Pflügers Archiv 467 1881-1898. (https://doi.org/10.1007/s00424-014-1619-7)

Weber MA, Mansfield TA, Cain VA, Iqbal N, Parikh S \& Ptaszynska A 2016 Blood pressure and glycaemic effects of dapagliflozin versus placebo in patients with type 2 diabetes on combination antihypertensive therapy: a randomised, double-blind, placebo-controlled, phase 3 study. Lancet Diabetes and Endocrinology 4 211-220. (https://doi. org/10.1016/S2213-8587(15)00417-9)

Young ME, Guthrie PH, Razeghi P, Leighton B, Abbasi S, Patil S, Youker KA \& Taegtmeyer H 2002 Impaired long-chain fatty acid oxidation and contractile dysfunction in the obese Zucker rat heart. Diabetes 51 2587-2595. (https://doi.org/10.2337/ diabetes.51.8.2587)

Zhou B, Lei S, Xue R, Leng Y, Xia Z \& Xia ZY 2017 DJ-1 overexpression restores ischaemic post-conditioning-mediated cardioprotection in diabetic rats: role of autophagy. Clinical Science 131 1161-1178. (https://doi.org/10.1042/CS20170052)

Received in final form 6 November 2017

Accepted 15 November 2017

Accepted Preprint published online 15 November 2017 http://joe.endocrinology-journals.org https://doi.org/10.1530/JOE-17-0457 (c) 2018 Society for Endocrinology Published by Bioscientifica Ltd. Printed in Great Britain 